University of Nebraska - Lincoln

DigitalCommons@University of Nebraska - Lincoln

Temporal coherent control of resonant two-photon double ionization of the hydrogen molecule via doubly excited states

Jean Marcel Ngoko Djiokap

Anthony F. Starace

Follow this and additional works at: https://digitalcommons.unl.edu/physicsstarace

Part of the Atomic, Molecular and Optical Physics Commons, Elementary Particles and Fields and String Theory Commons, and the Plasma and Beam Physics Commons

This Article is brought to you for free and open access by the Research Papers in Physics and Astronomy at DigitalCommons@University of Nebraska - Lincoln. It has been accepted for inclusion in Anthony F. Starace Publications by an authorized administrator of DigitalCommons@University of Nebraska - Lincoln. 


\title{
Temporal coherent control of resonant two-photon double ionization of the hydrogen molecule via doubly excited states
}

\author{
J. M. Ngoko Djiokap $\odot^{*}$ and Anthony F. Starace \\ Department of Physics and Astronomy, University of Nebraska, Lincoln, Nebraska 68588-0299, USA
}

(Received 23 October 2020; revised 24 March 2021; accepted 22 April 2021; published 11 May 2021)

\begin{abstract}
We use time-delayed, counter-rotating, circularly polarized few-cycle attosecond nonoverlapping pulses to study the temporal coherent control of the resonant process of two-photon double ionization (TPDI) of hydrogen molecule via doubly excited states for pulse propagation direction along $\hat{\mathbf{k}}$ either parallel or perpendicular to the molecular axis $\hat{\mathbf{R}}$. For $\hat{\mathbf{k}} \| \hat{\mathbf{R}}$ and a pulse carrier frequency of $36 \mathrm{eV}$ resonantly populating the $Q_{2}{ }^{1} \Pi_{u}^{+}(1)$ doubly excited state as well as other ${ }^{1} \Pi_{u}^{+}$doubly excited states, we find that the indirect ionization pathway through these doubly excited states changes the character of the kinematical vortex-shaped momentum distribution produced by the two direct ionization pathways from fourfold to twofold rotational symmetry. This result is similar to what found in TPDI of the He atom involving ${ }^{1} P_{ \pm 1}^{o}$ doubly excited states [Ngoko Djiokap and Starace, J. Opt. 19, 124003 (2017)]; however, angular distributions exhibiting a quantum beat effect between the ground state and a doubly excited state seen for the He atom are observed here for its molecular counterpart with an anomaly in shape and magnitude, not in frequency. The sixfold differential probability integrated over the azimuthal angle of the photoelectron pair shows that this anomaly is due to autoionization decays and quantum beats between doubly excited states. For $\hat{\mathbf{k}} \perp \hat{\mathbf{R}}$ and a broadband pulse carrier frequency of $30 \mathrm{eV}$ populating the $Q_{1}{ }^{1} \Pi_{u}^{+}(1)$, $Q_{1}{ }^{1} \Sigma_{u}^{+}(1), Q_{2}{ }^{1} \Pi_{u}^{+}(1)$, and $Q_{1}{ }^{1} \Sigma_{u}^{+}(2)$ doubly excited states, the momentum distribution is shown to exhibit dynamical electron vortices with four spiral arms, which originates from the interplay between the ${ }^{1} \Delta_{g}^{+},{ }^{1} \Pi_{g}^{+}$, and ${ }^{1} \Sigma_{g}^{+}$dynamical ionization amplitudes. Our treatment within either the adiabatic-nuclei approximation or fixed-nuclei approximation shows that the latter provides a very good account for this correlated process.
\end{abstract}

DOI: 10.1103/PhysRevA.103.053110

\section{INTRODUCTION}

Temporal coherent control in which a sequence of two time-delayed pulses is used to create and follow several quantum paths is an attractive way for achieving laser control of chemical and molecular processes [1-5]. For atomic ionization, while quantum interferences including three kinds of quantum beats could arise for both overlapping and nonoverlapping pulses, optical interference only occurs for overlapping pulses [5,6]. The first kind of quantum beats (called here QB-I) relies on the superposition between the ground state of energy $E_{g}$ and a bound state of energy $\epsilon_{k}$, which is established by the first pulse and has a beat frequency $\left(\epsilon_{k}-E_{g}\right)$. When the bandwidth of the pulses is broad enough to support several bound states coherently, the second kind of quantum beats (called QB-II) takes place and it relies on the population oscillation between the excited states with a period $2 \pi /\left(\epsilon_{k}-\epsilon_{k^{\prime}}\right)$ corresponding to the energy spacing between them. The third kind of quantum beats (called QB-III) of interest occurs when each quantum path leads to a continuum electron wave packet. Since the final continuum states have the same kinetic energy $E$, the created wave packets necessarily interfere when they overlap spatially and temporally at the detector, causing a modulation of the photoelectron signal due

\footnotetext{
${ }^{*}$ Corresponding author: marcelngoko@unl.edu
}

${ }^{\dagger}$ Deceased. to ambiguity in knowledge of which pulse was responsible for the birth of the electron. This QB-III, also known as Ramsey interference [7], can be perceived as a temporal analog to a Young's double-slit arrangement, which has a period of $2 \pi /\left(E+E_{b}\right)$, where $E_{b}$ is the binding energy of the system. While the period of QB-II is longer than that for QB-I, the latter is in turn longer than that for the QB-III; however, they may all occur simultaneously depending on the process and observable. Our primary interest is on the correlated process of two-photon transitions.

For the correlated process of a two-photon double ionization (TPDI) of the $\mathrm{He}$ atom by a pair of time-delayed linearly polarized attosecond pulses, several theoretical studies [8-13] have shown how the beat periods of the coherent superposition of intermediate states, both among themselves (QB-II) and with the ground state (QB-I), affects the $\mathrm{He}^{2+}$ yield. For a pair of nonoverlapping oppositely circularly polarized attosecond pulses, a recent theoretical study [14] based on the time-dependent second-order perturbation theory (PT) and full-dimensional time-dependent Schrödinger equation (TDSE) predicted the attosecond QB-III phenomenon in the angular distribution at a fixed excess energy $E=2 \omega+E_{g}$ for the nonresonant case of TPDI of the He atom, where four-arm spiral patterns were present in the two-electron momentum distribution. For the resonant case of TPDI of the $\mathrm{He}$ atom via doubly excited states, the indirect ionization path involving several doubly excited states was found to break down the fourfold rotational symmetry of the vortex-shaped 
momentum distribution. Also, QB-I involving the strongest $2 s 2 p^{1} P^{o}$ doubly excited state $[14,15]$ with a period of 69 attoseconds (as) was predicted in the angular distribution at a fixed excess energy $E$, in the energy-integrated angular distribution, and in the energy distribution [14]. For the attosecond time delays used in [14], QB-I involving other doubly excited states together with the rather slow (femtosecond) dynamical processes of autoionization $(\sim 17 \mathrm{fs})$ and QB-II between doubly excited states $(\sim 1.2 \mathrm{fs})$ were found to affect by only $8 \%$ the magnitude of angular distributions exhibiting this QB-I effect. As shown in this contribution, the possibility of extending such study for TPDI from the simplest two-electron atom to its molecular counterpart looks far from straightforward given its deviation from spherical symmetry, the rather steep repulsive potential curves of molecular doubly excited states with relatively short lifetimes and comparable oscillator strengths, and different electric dipole selection rules due to the molecular orientation $\hat{\mathbf{R}}$ with respect to the laser beam direction $\hat{\mathbf{k}}$. While TPDI of $\mathrm{H}_{2}$ by such attosecond pulse scheme has never been studied, our recent study of single-photon double ionization known as double photoionization (DPI) of fixed-in-space $\mathrm{H}_{2}$ [16] evidenced two types of electron vortices. For a particular class of electron detection geometry for which the mutual angle $\beta=\cos ^{-1}\left(\hat{\mathbf{p}}_{1} \cdot \hat{\mathbf{p}}_{2}\right)$ between the electron momenta $\mathbf{p}_{1}, \mathbf{p}_{2}$ is held fixed, while kinematical electron vortices similar to the one obtained in DPI of the He atom [17] were predicted in the momentum distribution for the $\hat{\mathbf{k}} \| \hat{\mathbf{R}}$ scheme, the occurrence of dynamical electron vortices were investigated for the $\hat{\mathbf{k}} \perp \hat{\mathbf{R}}$ scheme.

In this paper we use oppositely circularly polarized fewcycle attosecond nonoverlapping pulses to study the resonant process of TPDI of the hydrogen molecule isotopes via $Q_{1}$ and $Q_{2}$ doubly excited states. When those superexcited states are populated by one-photon absorption transition from the ${ }^{1} \Sigma_{g}^{+}$ground state by the first pulse, we study the occurrence of both kinematical and dynamical electron vortices, autoionization, as well as the three kinds of quantum beats. To allow comparison between TPDI of $\mathrm{He}$ and $\mathrm{H}_{2}$ initially in their ground states, we employ few-cycle pulses and time delays $\tau$ shorter than a femtosecond to freeze any nuclear dynamics between the electronic transitions involving these superexcited states with their rather steep repulsive potential curves. Our predictions for both the parallel [Fig. 1(a)] and perpendicular [Fig. 1(b)] pulse schemes are based on time-dependent fully $a b$ initio simulations within either the fixed-nuclei approximation [16,18-25] or the adiabatic-nuclei approximation [21]. Only for the $\hat{\mathbf{k}} \| \hat{\mathbf{R}}$ scheme in Fig. 1(a) that we extend the time-dependent second-order PT successfully applied for TPDI of $\mathrm{He}$ [14] to treat the resonant TPDI of $\mathrm{H}_{2}$.

Our findings are fourfold. First, for the $\hat{\mathbf{k}} \| \hat{\mathbf{R}}$ scheme in Fig. 1(a), a three-cycle pulse carrier frequency of $36 \mathrm{eV}$ is on resonance with the vertical transition at the equilibrium internuclear distance $R_{e}=1.4$ a.u. between the field-free ground state and $Q_{2}{ }^{1} \Pi_{u}^{+}(1)$ doubly excited state. Note that several doubly excited states, including the $Q_{1}{ }^{1} \Pi_{u}^{+}(1), Q_{1}{ }^{1} \Pi_{u}^{+}(2)$, $Q_{2}{ }^{1} \Pi_{u}^{+}(2)$, and $Q_{2}{ }^{1} \Pi_{u}^{+}(3)$ doubly excited states [26] with more or less comparable oscillator strength, are also populated thanks to the broad pulse bandwidth. We find that the indirect ionization pathway involving these doubly excited states changes the character of the kinematical vortex-shaped
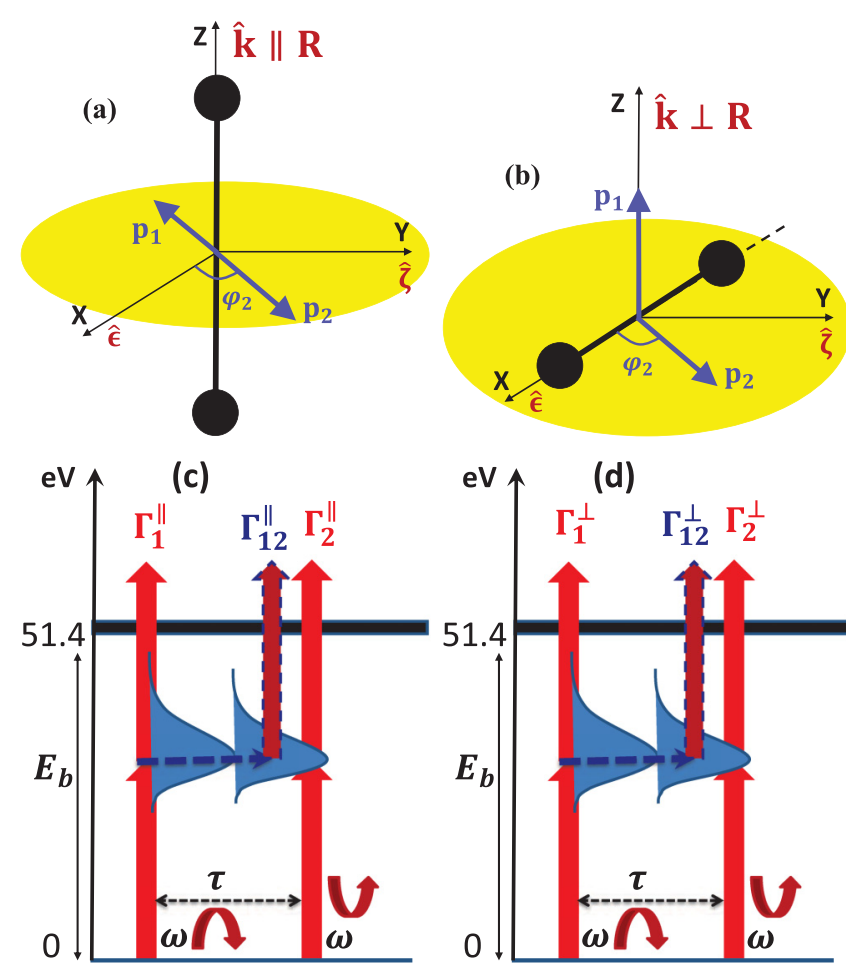

FIG. 1. Top: Detection geometries to study TPDI of fixed-inspace $T_{2}$ by nonoverlapping circularly polarized attosecond pulses propagating along $\hat{\mathbf{k}}$ either parallel (a) or perpendicular (b) to the molecular axis $\mathbf{R}$. In (a), $\mathbf{p}_{1}$ and $\mathbf{p}_{2}$ are emitted back-to-back (BTB) in the polarization $(x, y)$ plane; while in (b), $\mathbf{p}_{1} \| \hat{\mathbf{k}}$ while $\mathbf{p}_{2}$ is recorded in the polarization $(x, y)$ plane. For $\hat{\mathbf{k}} \| \mathbf{R}$ in (a), (c) shows three ionization paths: $\Gamma_{1}^{\|}$describes the $\Delta M=+1$ transitions ${ }^{1} \Sigma_{g}^{+} \rightarrow$ ${ }^{1} \Pi_{u}^{+}(M=+1) \rightarrow{ }^{1} \Delta_{g}^{+}(M=+2)$ for two-photon absorption by the RCP pulse from the ground state; $\Gamma_{2}^{\|}$describes the $\Delta M=-1$ transitions ${ }^{1} \Sigma_{g}^{+} \rightarrow{ }^{1} \Pi_{u}^{+}(M=-1) \rightarrow{ }^{1} \Delta_{g}^{+}(M=-2)$ for two-photon absorption by the LCP pulse (delayed in time by $\tau$ ) from the ground state; and $\Gamma_{12}^{\|}$describes the transitions ${ }^{1} \Sigma_{g}^{+} \rightarrow{ }^{1} \Pi_{u}^{+}(M=$ $+1) \rightarrow{ }^{1} \Sigma_{g}^{+}(M=0)$ for one-photon absorption by the RCP pulse from the ground state to doubly excited states, followed by onephoton absorption by the LCP pulse from doubly excited states. For $\hat{\mathbf{k}} \perp \mathbf{R}$ in (b), (d) shows three ionization pathways: $\Gamma_{1}^{\perp}$ describes the $\Delta M=+1,0$ transitions ${ }^{1} \Sigma_{g}^{+} \rightarrow\left[{ }^{1} \Pi_{u}^{+}(M=+1),{ }^{1} \Sigma_{u}^{+}(M=\right.$ $0)] \rightarrow\left[{ }^{1} \Delta_{g}^{+}(M=+2),{ }^{1} \Pi_{g}^{+}(M=+1)\right]$ for two-photon absorption by the RCP pulse from the ground state; $\Gamma_{2}^{\perp}$ describes the $\Delta M=-1,0$ transitions ${ }^{1} \Sigma_{g}^{+} \rightarrow\left[{ }^{1} \Pi_{u}^{+}(M=-1),{ }^{1} \Sigma_{u}^{+}(M=\right.$ $0)] \rightarrow\left[{ }^{1} \Delta_{g}^{+}(M=-2),{ }^{1} \Pi_{g}^{+}(M=-1)\right]$ for two-photon absorption by the LCP pulse from the ground state; and $\Gamma_{12}^{\perp}$ describes the transitions ${ }^{1} \Sigma_{g}^{+} \rightarrow\left[{ }^{1} \Pi_{u}^{+}(M=+1),{ }^{1} \Sigma_{u}^{+}(M=0)\right] \rightarrow$ $\left[{ }^{1} \Sigma_{g}^{+}(M=0),{ }^{1} \Pi_{g}^{+}(M=-1)\right]$ for one-photon absorption by the RCP pulse from the ground state and one-photon absorption by the LCP pulse from doubly excited states.

momentum distribution from fourfold to twofold symmetry. This result is similar to that found for $\mathrm{He}$ atom involving the strongest $2 s 2 p{ }^{1} P^{o}$ doubly excited state among the populated $2 \operatorname{sn} p{ }^{1} P^{o}$ doubly excited states [14].

Second, when varying $\tau$ for this $\hat{\mathbf{k}} \| \hat{\mathbf{R}}$ scheme within the fixed-nuclei approximation we find that the twofold symmetric angular distribution at a fixed excess energy $E$ rotates 
and exhibits the correct QB-I frequency associated with the $Q_{2}{ }^{1} \Pi_{u}^{+}(1)$ doubly excited state, but its shape and magnitude change with $\tau$. In contrast to He where a QB-I effect involving the strongest $2 s 2 p^{1} P^{o}$ doubly excited state was evidenced [14], it is found that this QB-I anomaly reported here in $\mathrm{H}_{2}$ at $R_{e}=1.4$ a.u. is not caused by the anisotropy (deviation from spherical symmetry) effect, due to electron-nucleus interactions in $\mathrm{H}_{2}$. Indeed, the anisotropy of the electron density is rather small at the equilibrium internuclear distance.

Third, by integrating the sixfold differential probability (SDP) over the azimuthal angle $\varphi$ of the photoelectron pair PT for this $\hat{\mathbf{k}} \| \hat{\mathbf{R}}$ scheme shows that any QB-I effect can be eliminated, isolating thus the two-electron dynamics due to autoionization and QB-II between doubly excited states. Our TDSE results for this observable reveal significant timedependent activities attributed to autoionization decays and QB-II, which mostly explain the QB-I anomaly seen for the $Q_{2}{ }^{1} \Pi_{u}^{+}(1)$ doubly excited state. Indeed, with an energy spacing of 2-8 eV between these doubly excited states [26] with rather comparable oscillator strengths and lifetimes shorter (by an order of magnitude) than that of the $2 s 2 p{ }^{1} P^{o}$ doubly excited state in $\mathrm{He}$, the QB-II effect with a period of $0.5-$ $2 \mathrm{fs}$ together with autoionization decays $(\sim 1.4 \mathrm{fs})$ in TPDI are expected to be more significant in $\mathrm{H}_{2}$ than in $\mathrm{He}$ for attosecond time delays.

Fourth, for $\hat{\mathbf{k}} \perp \hat{\mathbf{R}}$ in Fig. 1(b), a three-cycle pulse carrier frequency of $30 \mathrm{eV}$ is close to be resonant with the vertical transition at $R_{e}=1.4$ a.u. between the field-free ground state and the strongest $Q_{1}{ }^{1} \Sigma_{u}^{+}(1)$ or $Q_{2}{ }^{1} \Pi_{u}^{+}(1)$ doubly excited state. Note that several doubly excited states, including the $Q_{1}{ }^{1} \Sigma_{u}^{+}(2)$ and $Q_{2}{ }^{1} \Sigma_{u}^{+}(1)$ doubly excited states and the other states mentioned above, with more or less comparable oscillator strengths, are populated as well thanks to the broad pulse bandwidth. Despite those facts for $\hat{\mathbf{k}} \perp \hat{\mathbf{R}}$, we report here on dynamical electron vortices exhibiting four-arm spiral patterns in the momentum distribution since two photons are absorbed from each laser pulse. We show how this kind of electron vortices stems from an interplay between the ${ }^{1} \Delta_{g}^{+}$, ${ }^{1} \Pi_{g}^{+}$, and ${ }^{1} \Sigma_{g}^{+}$dynamical ionization amplitudes.

This paper is organized as followed. In Sec. II we present our numerical methods for solving the TDSE. In Sec. III PT is described and used to analyze our TDSE results in Secs. IV A, IV C, and IV D. In Secs. IV A and IV B we discuss effects of doubly excited states on momentum distributions for $\hat{\mathbf{k}} \| \mathbf{R}$ and $\hat{\mathbf{k}} \perp \mathbf{R}$, respectively. In Sec. IV C we discuss effects of doubly excited states on angular distributions for $\hat{\mathbf{k}} \| \mathbf{R}$. In Sec. IV D two-electron dynamics of autoionization and QB-II between doubly excited states are separated from any QB-I effect and discussed in the $\varphi$-integrated energy distribution for $\hat{\mathbf{k}} \| \mathbf{R}$. In Sec. $\mathrm{V}$ we summarize our results and draw some conclusions. Atomic units (a.u.) are used throughout this paper unless specified otherwise.

\section{NUMERICAL METHODS}

In this section we first provide a brief overview of our $a b$ initio computational methods for investigating ionization of hydrogen molecule by an arbitrarily polarized XUV light. Next, we describe our numerical procedures in obtaining the SDP within either the fixed-nuclei approximation or the adiabatic-nuclei approximation.

To obtain the SDP numerically, we have used our code that was developed and applied to investigate DPI of fixedin-space $\mathrm{H}_{2}$ by a pair of time-delayed elliptically polarized attosecond pulses [16]. It is an extension of our computer codes $[14,17,27-29]$ that were developed and used to investigate other ionization processes for the two-electron atomic system produced by elliptically polarized pulses, such as single ionization [28,29] and double ionization [14,17,27]. These codes are generalized to take into account the twocenter molecular hydrogen problem within the fixed-nuclei approximation (as in Refs. [16,18-25]). As in Refs. [18,22], we employ a single-center, time-dependent close-coupling expansion of the two-electron wave packet in spherical coordinates (with the origin at the center of mass of the nuclei). For a fixed internuclear separation $R$, the radial part of the two-electron wave packet $\Psi\left(\mathbf{r}_{1}, \mathbf{r}_{2} ; R, t\right)$ is discretized using a finite-element discrete variable representation (FE-DVR) [30]. As pointed out in Ref. [31], the FE-DVR scheme can be carefully chosen in order to avoid the singularities at $\mathbf{r}_{j}=$ $\pm \mathbf{R} / 2$, where $j=1,2$. To efficiently time propagate the wave packet $\Psi\left(\mathbf{r}_{1}, \mathbf{r}_{2} ; R, t\right)$ for elliptically polarized pulses, we use a real-space-product algorithm [31] together with a Wigner rotation transformation [32,33].

In our single-center approach in spherical coordinates, we first propagate the two-electron wave packet in imaginary time to prepare the initial ${ }^{1} \Sigma_{g}^{+}$ground state of the $\mathrm{H}_{2}$ molecule at a fixed $R$. In contrast to the He atom, the total electronic angular momentum $L$ is not a good quantum number for the hydrogen molecule. While the spherically symmetric He atom ground state has $L=0$, the nonspherical symmetry of the electron-nucleus interaction in the $\mathrm{H}_{2}$ molecule requires that the ground state is described using several even $L$ components. We include four total electronic angular momenta ( $L=0,2,4,6)$ and all combinations of individual electron orbital angular momenta $l_{1}, l_{2}=0-5$. Our calculated electronic energy of the ${ }^{1} \Sigma_{g}^{+}$ground state at the equilibrium internuclear separation $R_{e} \simeq 1.4$ a.u. is $E_{g}=-1.888573$ a.u. [16], which is to be compared with the benchmark value of -1.888760 [34] or -1.888761 a.u. [35], after taking out the internuclear repulsion.

Starting from the initial ${ }^{1} \Sigma_{g}^{+}$ground state at a fixed $R$, we solve the TDSE by including seven total electronic angular momenta $(L=0-6)$, their azimuthal quantum numbers $|M| \leqslant L$, all combinations of individual electron orbital angular momenta $l_{1}, l_{2}=0-5$, and their azimuthal quantum numbers $\left|m_{1}\right| \leqslant l_{1}$ and $\left|m_{2}\right| \leqslant l_{2}$. After the end of the pair of time-delayed circularly polarized pulses with total duration $T_{f}=\tau+T$ (where $T$ is the total duration of each pulse and $\tau$ is the time delay between the two laser pulses), we freely propagate in time the wave packet (solution of the TDSE) for a longer additional time $T_{p}$ in order to ensure that its doubly ionized part $\Psi_{C}\left(\mathbf{r}_{1}, \mathbf{r}_{2} ; R, t\right)$ is sufficiently far away from the nuclei, and also so that the two photoelectrons are well separated from each other $[18,19]$. At a time $t_{p}=T_{f}+T_{p}$, where $T_{p} \geqslant 35$ a.u., we obtain the SDP at $R=R_{e}$ within the fixed-nuclei approximation

$$
\mathcal{W}\left(\mathbf{p}_{1}, \mathbf{p}_{2} ; R_{e}\right)=\left|A\left(\mathbf{p}_{1}, \mathbf{p}_{2} ; R_{e}\right)\right|^{2},
$$


from the TPDI amplitude

$$
A\left(\mathbf{p}_{1}, \mathbf{p}_{2} ; R\right)=\left\langle\Phi_{\mathbf{p}_{1}, \mathbf{p}_{2}}^{(-)}\left(\mathbf{r}_{1}, \mathbf{r}_{2}\right) \mid \Psi_{C}\left(\mathbf{r}_{1}, \mathbf{r}_{2} ; R, t_{p}\right)\right\rangle,
$$

where $\Phi_{\mathbf{p}_{1}, \mathbf{p}_{2}}^{(-)}$is the field-free double-continuum final state with excess energy $E=\left(p_{1}^{2}+p_{2}^{2}\right) / 2$, which is approximated by a product of two Coulomb waves with charge $Z=2$ $[18,19]$. In the projection (3) we note that $\Psi_{C}\left(\mathbf{r}_{1}, \mathbf{r}_{2} ; R, t\right)$ equals the wave packet (that we obtain by solving the TDSE within a radial box of 350 a.u.) from which we have removed contributions of bound and singly ionized states (as done in Refs. [18,36]).

The derivation of the TPDI probability (1) implicitly relies on the Born-Oppenheimer approximation, where the electronic and nuclear motions are separated. Using sufficiently short (attosecond) pulses and time delays, we assume there is no nuclear dynamics between the electronic transitions. When nuclear motion is considered in both the initial and final states, we treat the process of TPDI of the hydrogen molecule within the adiabatic-nuclei approximation by using the so-called Chase's approximation to obtain the ionization amplitude, as in Ref. [21]:

$$
A_{\kappa}\left(\mathbf{p}_{1}, \mathbf{p}_{2}\right)=\int_{0}^{\infty} d R \chi_{\kappa}(R) A\left(\mathbf{p}_{1}, \mathbf{p}_{2} ; R\right) \chi_{0}(R),
$$

where $A\left(\mathbf{p}_{1}, \mathbf{p}_{2} ; R\right)$ is defined by Eq. (2). We have used the axial approximation, which assumes that following absorption of two photons from an ultrashort laser pulse, the nuclei fly apart along a vector that remains stationary in the laboratory frame. Here $\chi_{0}(R)$ is the initial-state vibrational wave function, which within the harmonic-oscillator approximation, writes

$$
\chi_{0}(R)=\left(\sqrt{\mu \omega_{0} / \pi}\right)^{1 / 2} e^{-\mu \omega_{0}\left(R-R_{e}\right)^{2} / 2},
$$

where $\omega_{0} / 2=\sqrt{k_{m} / 4 \mu}$ is the zero-point energy, with $k_{m}$ and $\mu$ being the force constant and reduced mass of the molecular isotope, respectively. Physically, this zero-point energy raises the energy for the ground state of nuclear motion above the minimum in the potential curve $U(R)$, so that the equilibrium dissociation energy $D_{e}$ and the groundvibrational-state dissociation energy $D_{0}$ differ by $\omega_{0} / 2$. The measured zero-point energy is $0.27284 \mathrm{eV}$ for $\mathrm{H}_{2}$ [37] with $\mu\left(H_{2}\right)=1836 / 2,0.193135 \mathrm{eV}$ for $\mathrm{D}_{2}$ [37] with $\mu\left(D_{2}\right)=$ $2 \mu\left(H_{2}\right)$, and $0.15278 \mathrm{eV}$ for $\mathrm{T}_{2}$ [38] with $\mu\left(T_{2}\right)=3 \mu\left(H_{2}\right)$, meaning that the vibrational broadening due to the quantum uncertainty relation (zero-point energy) is smallest for $T_{2}$. Knowing these two physical quantities, the calculated force constants $k_{m}$ for these three isotopes are respectively 574.79 , 576.03 , and $540.69 \mathrm{~N} / \mathrm{m}$. The first two values for $k_{m}$ have to be compared with those given in Table 3.3 from Ref. [39], which are $573.4 \mathrm{~N} / \mathrm{m}$ for $\mathrm{H}_{2}$ and $576.9 \mathrm{~N} / \mathrm{m}$ for $\mathrm{D}_{2}$.

For the final state of our dissociative TPDI process, coherent electronic and nuclear wave packets are created with different energy sharing between these two degrees of freedom. The final-state nuclear wave function $\chi_{\kappa}(R)$ satisfying

$$
\left(-\frac{1}{2 \mu} \frac{d^{2}}{d R^{2}}+\frac{1}{R}-\frac{\kappa^{2}}{2 \mu}\right) \chi_{\kappa}(R)=0
$$

is simply the repulsive Coulomb function corresponding to two bare protons. Given the broad pulse bandwidth $\Delta \omega$, the energy conservation relation writes $E_{0}+2 \omega \pm 2 \Delta \omega=$ $E+E_{N}$, where $E=E_{1}+E_{2}$ is the excess energy shared by electrons, $E_{N}=\kappa^{2} / 2 \mu$ is the nuclear kinetic energy release (KER), and $E_{0}=-1.888761$ a.u. $+1 / R_{e}+\omega_{0} / 2$ is the initial target energy of the hydrogen molecular isotope.

In evaluating the TPDI amplitude (3), we note that the lower and upper classical turning points of the molecule in its initial vibrational state, located, respectively, at $R_{<} \simeq 1.0$ a.u. and $R_{>} \simeq 1.9$ a.u., place effective upper and lower bounds on the observable of KER. For a carrier frequency $\omega$ so that $2 \omega$ is well above the Franck-Condon threshold, the nuclear wave function $\chi_{\kappa}(R)$ oscillates rapidly beyond its classical turning point $R_{c}$, which necessarily lies within the Frank-Condon interval $\left[R_{<}, R_{>}\right]$over the range of observable KER. In such cases, the reflection approximation can be used to describe $\chi_{\kappa}(R)$ by a delta function at the classical turning point $R_{c}$,

$$
\chi_{\kappa}(R)=N_{\kappa} \delta\left(R-R_{c}\right),
$$

where there is a one-to-one mapping between the KER and the internuclear distance, i.e., $E_{N}=1 / R_{c}$. Using the energynormalization condition, one can determine the normalization constant $N_{\kappa}=|d U / d R|_{R=R_{c}}^{-1 / 2}$, which involves the slope of the final-state potential curve. Since $U(R)=1 / R$ in the final state, therefore $N_{\kappa}=R_{c}$. Substituting $\chi_{\kappa}(R)(6)$ in $A_{\kappa}\left(\mathbf{p}_{1}, \mathbf{p}_{2}\right)$ (3), and integrating over the internuclear separation $R$, leads to

$$
A_{\kappa}\left(\mathbf{p}_{1}, \mathbf{p}_{2}\right)=R_{c} A\left(\mathbf{p}_{1}, \mathbf{p}_{2} ; R_{c}\right) \chi_{0}\left(R_{c}\right) .
$$

Therefore, taking the squared modulus of the amplitude (7) gives a sevenfold differential probability (with respect to the electron momenta $\mathbf{p}_{1}, \mathbf{p}_{2}$ and the nuclear KER $E_{N}$ ) for our process of TPDI:

$$
\mathcal{W}_{\kappa}\left(\mathbf{p}_{1}, \mathbf{p}_{2}\right)=R_{c}^{2}\left|A\left(\mathbf{p}_{1}, \mathbf{p}_{2} ; R_{c}\right) \chi_{0}\left(R_{c}\right)\right|^{2} .
$$

Integrating this sevenfold differential probability over the $\mathrm{KER}, E_{N}=1 / R_{c}$, i.e., over $R_{c}$, yields the SDP:

$$
\mathcal{W}\left(\mathbf{p}_{1}, \mathbf{p}_{2}\right)=\int d R_{c} \mathcal{W}\left(\mathbf{p}_{1}, \mathbf{p}_{2} ; R_{c}\right)\left|\chi_{0}\left(R_{c}\right)\right|^{2},
$$

where $\mathcal{W}\left(\mathbf{p}_{1}, \mathbf{p}_{2} ; R_{c}\right)$ is defined as in Eq. (1) by replacing $R_{e}$ by $R_{c}$. Finally, since the Gaussian-shaped $\chi_{0}(R)$ [see, e.g., Eq. (4)] is strongly peaked about the equilibrium internuclear distance $R_{e}$, one may replace $\mathcal{W}\left(\mathbf{p}_{1}, \mathbf{p}_{2} ; R_{c}\right)$ by $\mathcal{W}\left(\mathbf{p}_{1}, \mathbf{p}_{2} ; R_{e}\right)$ and take it outside of the integral (9):

$$
\mathcal{W}\left(\mathbf{p}_{1}, \mathbf{p}_{2}\right)=\mathcal{W}\left(\mathbf{p}_{1}, \mathbf{p}_{2} ; R_{e}\right) \int d R_{c}\left|\chi_{0}\left(R_{c}\right)\right|^{2} .
$$

Given that the initial-state vibrational wave function $\chi_{0}(R)$ is normalized to unity, the SDP (10) within the adiabaticnuclei approximation under such a reasonable consideration naturally reduces to the SDP within the fixed-nuclei approximation defined by Eq. (1). Below in Sec. IV, we test the validity of the fixed-nuclei approximation compared to the more realistic adiabatic-nuclei approximation. As in Ref. [16], TDSE results for momentum, angular, and energy distributions presented in Sec. IV have been verified to be converged. 
TABLE I. Position $\epsilon_{k}(\mathrm{eV})$ with respect to the minimum of the ground-state energy curve $U(R)$, width $\Gamma_{k}(\mathrm{eV})$, lifetime $\tau_{k}$ (fs), and transition dipole moment $\mu_{k}$ (a.u.) of some relevant doubly excited autoionizing states of $\mathrm{H}_{2}$ as a function of the internuclear distance $R$.

\begin{tabular}{lcllcl}
\hline \hline & $R$ (a.u.) & $\epsilon_{k}[26]$ & $\Gamma_{k}[26]$ & $\tau_{k}[26]$ & $\mu_{k}[40]$ \\
\hline$Q_{1}{ }^{1} \Pi_{u}^{+}(1)$ & 1.0 & 40.3 & 0.033 & 19.9 & 0.0584 \\
& 1.2 & 36.9 & 0.0096 & 68.2 & 0.03567 \\
& 1.4 & $\mathbf{3 4 . 2}$ & 0.0053 & $\mathbf{1 2 4 . 1}$ & $\mathbf{0 . 0 3 5 0 5}$ \\
& 1.6 & 31.8 & 0.0069 & 94.9 & 0.03145 \\
$Q_{1}{ }^{1} \Pi_{u}^{+}(2)$ & 1.4 & 34.9 & 0.0038 & 172.9 & \\
$Q_{2}{ }^{1} \Pi_{u}^{+}(1)$ & 1.0 & 39.8 & 0.05 & 12.4 & 0.05998 \\
& 1.2 & 37.9 & 0.033 & 20.08 & 0.06319 \\
& 1.4 & $\mathbf{3 6 . 3}$ & 0.481 & $\mathbf{1 . 3 7}$ & $\mathbf{0 . 0 6 4 4}$ \\
& 1.6 & 34.8 & 0.386 & 1.7 & 0.06506 \\
& 1.8 & 33.6 & 0.32 & 2.1 & 0.06060 \\
$Q_{2}{ }^{1} \Pi_{u}^{+}(2)$ & 1.4 & 38.3 & 0.131 & 5.0 & \\
$Q_{2}{ }^{1} \Pi_{u}^{+}(3)$ & 1.4 & 38.5 & 0.017 & 38.7 & \\
$Q_{1}{ }^{1} \Sigma_{u}^{+}(1)$ & 1.0 & 37.5 & 0.246 & 2.67 & 0.07930 \\
& 1.2 & 34.6 & 0.33 & 1.97 & 0.08823 \\
& 1.4 & $\mathbf{3 1 . 9 9}$ & 0.427 & $\mathbf{1 . 5 4}$ & $\mathbf{0 . 0 9 5 7 1}$ \\
& 1.6 & 29.7 & 0.515 & 1.28 & 0.1012 \\
$Q_{1}{ }^{1} \Sigma_{u}^{+}(2)$ & 1.0 & 39.88 & 0.055 & 12.0 & 0.03277 \\
& 1.2 & 36.85 & 0.0776 & 8.5 & 0.03707 \\
& 1.4 & $\mathbf{3 4 . 1 4}$ & 0.104 & $\mathbf{6 . 3}$ & $\mathbf{0 . 0 4 0 6 3}$ \\
& 1.6 & 31.75 & 0.129 & 5.1 & 0.04162 \\
$Q_{2}{ }^{1} \Sigma_{u}^{+}(1)$ & 1.4 & 38.58 & 0.011 & 60.5 & \\
$Q_{2}{ }^{1} \Sigma_{u}^{+}(2)$ & 1.4 & 39.19 & 0.006 & 115.2 & \\
\hline \hline & & & & & \\
\hline
\end{tabular}

\section{PERTURBATION THEORY ANALYSIS}

To describe and analyze the resonant process of TPDI of the fixed-in-space hydrogen molecules where $\hat{\mathbf{k}} \| \mathbf{R}$, below we generalize the PT analysis that was developed and successfully applied to treat TPDI of He [14]. As for He atom, the use of circularly polarized pulses for achieving the temporal coherent control of resonant TPDI of its molecular counterpart via doubly excited states strongly depends upon the pulse parameters. As shown below, we choose pulse parameters and isotopes to allow comparison between TPDI of hydrogen molecule within the fixed-nuclei approximation and helium atom. First, we choose the carrier frequency $\omega \simeq 36 \mathrm{eV}$ of the two pulses such that it is on resonance with the transition between the electronic ground state and the $Q_{2}{ }^{1} \Pi_{u}^{+}(1)$ doubly excited state at the equilibrium internuclear distance $R_{e} \simeq 1.4$, see Table I. Second, the bandwidth $\Delta \omega$ of the first pulse should be broad enough to support spiral patterns (Ramsey dark and bright interference fringes) in the momentum distribution. However, it should not be so broad as to allow double ionization of $\mathrm{H}_{2}$ (with a ground state binding energy at $R=R_{e}$ of $51.4 \mathrm{eV}$ ) by a single-photon transition.

Third, sufficiently short pulses should be used to make the molecular TPDI problem more atomiclike by freezing the nuclear motion between the electron transitions. Although this frozen-nuclei approximation avoids a complete mess in the time-development of different wave packets, it allows for different electronic components and thus beat frequencies. Furthermore, using Newton's second law to estimate the minimum time $\tau_{d}$ it takes the two nuclei initially at rest to move apart by $\Delta R=0.1$ a.u. due to the rather steep repulsive potential curves of the $Q_{1}$ or $Q_{2}$ autoionizing states, we find $\tau_{d}=(2 \mu \Delta R / F)^{1 / 2}$. Extracting the force $F=-\nabla U$ from the repulsive potential curves $U(R)$ of the $Q_{1}\left(Q_{2}\right)$ autoionizing states [41], we obtain $\tau_{d} \simeq 474$ as (541 as) for $\mathrm{H}_{2}, 670$ as (765 as) for $\mathrm{D}_{2}$, and 821 as (938 as) for $\mathrm{T}_{2}$. Therefore, for few-cycle pulses with duration less than 500 as and time delays $\tau$ shorter than its upper bound $\tau_{d}$, the frozen-nuclei approximation with atomiclike transitions for these isotopes can be adopted to freeze any nuclear dynamics involving the $Q_{1}$ or $Q_{2}$ doubly excited states.

When choosing each pulse with $n=3$ cycles corresponding to a duration $T \simeq 345$ as, the pulse bandwidth is $\Delta \omega \simeq$ $1.44 \omega / n=17.28 \mathrm{eV}$ for a cosine-squared envelope, meaning that all these conditions are well fulfilled. At this resonant carrier frequency and with such large $\Delta \omega$, Table I shows that not only the $Q_{1}$ doubly excited states but also the $Q_{2}$ doubly excited states are populated. Some examples of such states listed in Table I include the $Q_{1}{ }^{1} \Pi_{u}^{+}(1), Q_{1}{ }^{1} \Pi_{u}^{+}(2), Q_{2}{ }^{1} \Pi_{u}^{+}(2)$, and $Q_{2}{ }^{1} \Pi_{u}^{+}(3)$ doubly excited states, with the transition dipole moment $\mu_{k}$ of the first state being comparable to that of the $Q_{2}{ }^{1} \Pi_{u}^{+}(1)$ doubly excited state. Thus, the two-electron wave packet created by the first pulse encompasses several doubly excited states, with possibility of occurrence of quantum beat phenomena. Fourth, the first pulse has a peak intensity of $50 \mathrm{TW} / \mathrm{cm}^{2}$ with a maximum of $100 \mathrm{TW} / \mathrm{cm}^{2}$, which are not very intense such that higher-order nonlinear effects beyond the second-order ones occur. These intensities are strong enough to transfer significant population into these doubly excited states by one-photon transition from the ground state. Also, for such pulse intensity the so-called rotating wave approximation (RWA) is valid meaning that photon emission processes are negligible.

For pulse parameters used here, the Keldysh parameter is $\gamma \simeq 46$ and the ponderomotive energy is $U_{p} \simeq 12 \mathrm{meV}$, which is much smaller than $\omega$. Thus, PT analysis is valid and is then used to analyze our numerical TDSE results. In the following, for only the case where $\hat{\mathbf{k}} \| \hat{\mathbf{R}}$, we parametrize the PT amplitude and probability for TPDI of the hydrogen molecule in terms of the vectors of the problem, namely, the pulse polarization vector $\mathbf{e}$, and the photoelectron momenta $\mathbf{p}_{1}, \mathbf{p}_{2}$. The pulse polarization vector $\mathbf{e}$ is defined as

$$
\mathbf{e}=(\hat{\boldsymbol{\epsilon}}+i \eta \hat{\zeta}) / \sqrt{1+\eta^{2}},
$$

where $\eta$ is the pulse ellipticity, and $\hat{\boldsymbol{\epsilon}}$ and $\hat{\zeta}=[\hat{\mathbf{k}} \times \hat{\boldsymbol{\epsilon}}]$ are the major and minor axes of the polarization ellipse. Note that $\eta=+1$ for a right-circularly polarized (RCP) pulse, $\eta=-1$ for a left-circularly polarized (LCP) pulse.

From symmetry properties of the atomic and molecular problems, it can be shown that the parametrization of the PT amplitude for our TPDI molecular process when $\hat{\mathbf{k}} \| \mathbf{R}$ and for our TPDI atomic process of He have the same vectorial structure. Thus, the parametrization for our TPDI molecular process described below is just an extension of that for the TPDI atomic process of the He [14]. Although the PT developed in Ref. [14] was for the specific detection geometry of in-plane back-to-back (BTB) emission of photoelectrons, $\hat{\mathbf{p}}_{2}=-\hat{\mathbf{p}}_{1}$, as in Fig. 1(a), it also applies for the case of orthogonal detection geometry where the electron momentum $\mathbf{p}_{1}$ is emitted along the pulse propagation direction $\hat{\mathbf{k}}$ and the 
other electron momentum $\mathbf{p}_{2}$ is recorded in the polarization plane. For these two detection geometries and under the RWA and fixed-nuclei approximation, the PT amplitude for TPDI of hydrogen molecule by two nonoverlapping counter-rotating circularly polarized attosecond pulses delayed in time by $\tau$ can thus be written in the same form as Eq. (5) in Ref. [14]:

$$
A\left(\mathbf{p}_{1}, \mathbf{p}_{2} ; R_{e}\right)=e^{-2 i \phi_{1}}\left[A_{\Gamma_{1}}+A_{\Gamma_{2}}+A_{\Gamma_{12}}\right],
$$

where $\phi_{1}$ is the carrier-envelope phase (CEP) of the first pulse; $\hat{\mathbf{p}} \equiv \hat{\mathbf{p}}_{2}$ for the two detection geometries; and

$$
A_{\Gamma_{1}}=\mathcal{A}_{\Delta}(\rho)(\hat{\mathbf{p}} \cdot \mathbf{e})^{2}
$$

describes the transitions ${ }^{1} \Sigma_{g}^{+} \rightarrow{ }^{1} \Pi_{u}^{+}(M=+1) \rightarrow$ ${ }^{1} \Delta_{g}^{+}(M=+2)$ for two-photon absorption by the first RCP pulse from the ground state to the double continua,

$$
A_{\Gamma_{2}} \simeq \mathcal{A}_{\Delta}(\rho)\left(\hat{\mathbf{p}} \cdot \mathbf{e}^{*}\right)^{2} e^{i \Phi}
$$

describes the transitions ${ }^{1} \Sigma_{g}^{+} \rightarrow{ }^{1} \Pi_{u}^{+}(M=-1) \rightarrow$ ${ }^{1} \Delta_{g}^{+}(M=-2)$ for two-photon absorption by the timedelayed second LCP pulse from essentially the same ground state to the double continua, and

$$
A_{\Gamma_{12}}=\left[\mathcal{B}_{\Sigma}(\rho ; \tau)|\hat{\mathbf{p}} \cdot \mathbf{e}|^{2}+\mathcal{C}_{\Sigma}(\rho ; \tau)\right] e^{i \psi}
$$

describes the transitions ${ }^{1} \Sigma_{g}^{+} \rightarrow{ }^{1} \Pi_{u}^{+}(M=+1) \rightarrow$ ${ }^{1} \Sigma_{g}^{+}(M=0)$ for one-photon absorption by the first RCP pulse from the ground state to doubly excited states, followed by one-photon absorption by the second LCP pulse from doubly excited states to the double continua. In Eqs. (13)-(15) the argument $\rho$ of the dynamical parameters $\mathcal{A}_{\Delta}, \mathcal{B}_{\Sigma}$, and $\mathcal{C}_{\Sigma}$ is defined as $\rho \equiv\left(p_{1}, p_{2}, u, u_{1}, u_{2}\right)$, where $u=\hat{\mathbf{p}}_{1} \cdot \hat{\mathbf{p}}_{2}$, $u_{1}=\hat{\mathbf{p}}_{1} \cdot \hat{\mathbf{R}}$, and $u_{2}=\hat{\mathbf{p}}_{1} \cdot \hat{\mathbf{R}}$. Parity conservation and particle exchange rules require that the dynamical parameters $\mathcal{A}_{\Delta}(\rho)$, $\mathcal{B}_{\Sigma}(\rho ; \tau)$, and $\mathcal{C}_{\Sigma}(\rho ; \tau)$ are symmetric under the exchange of electron momenta $\mathbf{p}_{1} \leftrightarrow \mathbf{p}_{2}$. In Eq. (14), the Ramsey phase $\Phi$ defined as

$$
\Phi=\left(E-E_{g}\right) \tau+2 \phi_{12}
$$

is comprised of two terms: $\left(E-E_{g}\right) \tau$ is the difference in the phase accumulated by the pair of two-electron wave packets produced with a time delay $\tau$ via the two direct paths $\Gamma_{1}^{\|}$and $\Gamma_{2}^{\|}$, and $\phi_{12}$ is the relative CEP between the two pulses. In Eq. (15) the phase $\psi$ defined as

$$
\psi=E \tau+\phi_{12}
$$

expresses the fact that when the two-electron wave packet is created via the path $\Gamma_{12}^{\|}$, the two-electron wave packet created via the path $\Gamma_{1}^{\|}$has already acquired a phase $-E \tau$. Using Eq. (11), the geometric factors $(\hat{\mathbf{p}} \cdot \mathbf{e})^{2}$ and $|\hat{\mathbf{p}} \cdot \mathbf{e}|^{2}$ in Eqs. (13)-(15) expressed in terms of the spherical angles $(\theta, \varphi)$ of the electron momentum $\mathbf{p}$,

$$
\begin{gathered}
(\hat{\mathbf{p}} \cdot \mathbf{e})^{2}=\frac{1}{2} \sin ^{2} \theta e^{ \pm 2 i \varphi} \\
|\hat{\mathbf{p}} \cdot \mathbf{e}|^{2}=\frac{1}{2} \sin ^{2} \theta
\end{gathered}
$$

allow us to better interpret each of the three dynamical parameters $\mathcal{A}_{\Delta}(\rho), \mathcal{B}_{\Sigma}(\rho ; \tau)$, and $\mathcal{C}_{\Sigma}(\rho ; \tau)$. Each of these $\varphi$-independent molecular dynamical parameters is a product of the Fourier transform of the pulse envelope and the transition matrix element between the initial ${ }^{1} \Sigma_{g}^{+}(M=0)$ ground state and a final continuum state. While the final state is the ${ }^{1} \Delta_{g}^{+}(M= \pm 2)$ continuum states for $\mathcal{A}_{\Delta}(\rho)$, it is the ${ }^{1} \Sigma_{g}^{+}(M=0)$ continuum states with even values of $L$ for $\mathcal{B}_{\Sigma}(\rho ; \tau)$ and with only $L=0$ for $\mathcal{C}_{\Sigma}(\rho ; \tau)$.

Taking the squared modulus of the PT amplitude (12), we obtain the SDP for TPDI of the hydrogen molecules by a pair of nonoverlapping oppositely circularly polarized attosecond pulses for $\hat{\mathbf{k}} \| \mathbf{R}$ and for the two detection geometries,

$$
\begin{aligned}
\mathcal{W}\left(\mathbf{p}_{1}, \mathbf{p}_{2} ; R_{e}\right)= & \left|A_{\Gamma_{1}}+A_{\Gamma_{2}}\right|^{2}+\left|A_{\Gamma_{12}}\right|^{2} \\
& +2 \operatorname{Re}\left[A_{\Gamma_{1}}^{*} A_{\Gamma_{12}}\right]+2 \operatorname{Re}\left[A_{\Gamma_{2}}^{*} A_{\Gamma_{12}}\right],
\end{aligned}
$$

which contains four terms. Substituting the geometric factor (18) in Eqs. (13) and (14), the first term in the SDP (20) describing Ramsey interference between these two direct ionization pathways $\Gamma_{1}^{\|}$and $\Gamma_{2}^{\|}$writes

$$
\left|A_{\Gamma_{1}}+A_{\Gamma_{2}}\right|^{2}=\left|\mathcal{A}_{\Delta}(\rho)\right|^{2} \sin ^{4} \theta \cos ^{2}(\Phi / 2-2 \xi \varphi),
$$

where $\xi=+1(-1)$ for RCP/LCP (LCP/RCP) pulses.

The second term $\left|A_{\Gamma_{12}}\right|^{2} \equiv\left|A_{\Gamma_{12}}(\rho, \theta, \tau)\right|^{2}$ in the $\operatorname{SDP}(20)$ describes the pump-probe transition. Moreover, the form of the geometric factor (19) show that this second term does not depend on the azimuthal angle $\varphi$ of the electron momentum unit vector $\hat{\mathbf{p}}$, but does depend on both the polar angle $\theta$ and time delay $\tau$. Using the Hermitian stationary Green's function in the second-order PT amplitude, $A_{\Gamma_{12}}(\rho, \theta, \tau)$ can be approximated as a sum over intermediate autoionizing (doubly excited) states in which we introduce the decay factors $e^{-\tau / \tau_{k}}$ :

$$
A_{\Gamma_{12}}(\rho, \theta, \tau)=\sum_{k} e^{-i \epsilon_{k} \tau} A_{\Gamma_{12}}^{k}(\rho, \theta) e^{-\tau / \tau_{k}},
$$

where $k$ runs from 1 to $N$, with $N$ being the number of doubly excited states (with energy $\epsilon_{k}$ and lifetime $\tau_{k}$ ) lying within the bandwidth of the pump pulse. Using Eq. (22), the pump-probe signal $\left|A_{\Gamma_{12}}(\rho, \theta, \tau)\right|^{2}$ becomes

$$
\begin{aligned}
\left|A_{\Gamma_{12}}(\rho, \theta, \tau)\right|^{2}= & \sum_{k}\left|A_{\Gamma_{12}}^{k}(\rho, \theta)\right|^{2} e^{-2 \tau / \tau_{k}} \\
& +2 \sum_{k} \sum_{k^{\prime}>k}\left|A_{\Gamma_{12}}^{k *}(\rho, \theta)\right|\left|A_{\Gamma_{12}}^{k^{\prime}}(\rho, \theta)\right| \\
& \times e^{-\tau / \tau_{k}} e^{-\tau / \tau_{k^{\prime}}} \\
& \times \cos \left[\left(\gamma_{k^{\prime}}-\gamma_{k}\right)+\left(\epsilon_{k}-\epsilon_{k^{\prime}}\right) \tau\right],
\end{aligned}
$$

where $\gamma_{k} \equiv \gamma_{k}(\rho, \theta)$ is the phase of the dynamical parameter $A_{\Gamma_{12}}^{k}(\rho, \theta)$.

The third term in the SDP (20), which describes interference between the pathways $\Gamma_{1}^{\|}$and $\Gamma_{12}^{\|}$, writes

$$
\begin{aligned}
2 \operatorname{Re}\left[A_{\Gamma_{1}}^{*} A_{\Gamma_{12}}\right]= & 2\left|\mathcal{A}_{\Delta}^{*}(\rho) A_{\Gamma_{12}}(\rho, \theta, \tau)\right| \sin ^{2} \theta \\
& \times \cos ^{2}\left\{\left[E \tau+\phi_{12}+\Theta(\rho, \theta, \tau)\right] / 2-\xi \varphi\right\},
\end{aligned}
$$

while the fourth term in the SDP (20), which describes interference between the pathways $\Gamma_{2}^{\|}$and $\Gamma_{12}^{\|}$, writes

$$
\begin{aligned}
2 \operatorname{Re}\left[A_{\Gamma_{2}}^{*} A_{\Gamma_{12}}\right]= & -2\left|\mathcal{A}_{\Delta}^{*}(\rho) A_{\Gamma_{12}}(\rho, \theta, \tau)\right| \sin ^{2} \theta \\
& \times \sin ^{2}\left\{\left[E_{g} \tau-\phi_{12}+\Theta(\rho, \theta, \tau)\right] / 2+\xi \varphi\right\} .
\end{aligned}
$$


In Eqs. (24) and (25) we note the presence of a dynamical phase $\Theta(\rho, \theta, \tau)$ defined by

$$
\tan \Theta(\rho, \theta, \tau)=\frac{\operatorname{Im}\left[\mathcal{A}_{\Delta}^{*}(\rho) A_{\Gamma_{12}}(\rho, \theta, \tau)\right]}{\operatorname{Re}\left[\mathcal{A}_{\Delta}^{*}(\rho) A_{\Gamma_{12}}(\rho, \theta, \tau)\right]},
$$

which is the phase of the dynamical parameter $\mathcal{A}_{\Delta}^{*}(\rho) A_{\Gamma_{12}}(\rho, \theta, \tau)$, whose squared modulus writes

$$
\begin{aligned}
& \left|\mathcal{A}_{\Delta}^{*}(\rho) A_{\Gamma_{12}}(\rho, \theta, \tau)\right|^{2} \\
& =\sum_{k}\left|\mathcal{A}_{\Delta}^{*}(\rho) A_{\Gamma_{12}}^{k}(\rho, \theta)\right|^{2} e^{-2 \tau / \tau_{k}} \\
& \quad+2 \sum_{k} \sum_{k^{\prime}>k}\left|\mathcal{A}_{\Delta}(\rho) A_{\Gamma_{12}}^{k *}(\rho, \theta)\right|\left|\mathcal{A}_{\Delta}^{*}(\rho) A_{\Gamma_{12}}^{k^{\prime}}(\rho, \theta)\right| \\
& \quad \times e^{-\tau / \tau_{k}} e^{-\tau / \tau_{k^{\prime}}} \cos \left[\left(\alpha_{k^{\prime}}-\alpha_{k}\right)+\left(\epsilon_{k}-\epsilon_{k^{\prime}}\right) \tau\right], \quad
\end{aligned}
$$

where $\alpha_{k} \equiv \alpha_{k}(\rho, \theta)$ is the phase of the dynamical parameter $\mathcal{A}_{\Delta}^{*}(\rho) A_{\Gamma_{12}}^{k}(\rho, \theta)$.

An analysis of the term $\left|\mathcal{A}_{\Delta}^{*}(\rho) A_{\Gamma_{12}}(\rho, \theta, \tau)\right|$ (27) present in Eqs. (24) and (25) shows that autoionization decays with rates $1 / \tau_{k}$ and QB-II processes between doubly excited states with frequencies $\left(\epsilon_{k}-\epsilon_{k^{\prime}}\right)$ may occur thanks to the interferences between the direct pathway $\Gamma_{1}^{\|}$or $\Gamma_{2}^{\|}$with the indirect pathway $\Gamma_{12}^{\|}$. Also, these two time-dependent phenomena are present in the pump-probe term $\left|A_{\Gamma_{12}}(\rho, \theta, \tau)\right|^{2}$ (23). For time delays $\tau$ shorter than a femtosecond used in this work, one expects autoionization decays of the $Q_{2}{ }^{1} \Pi_{u}^{+}(1)$ doubly excited state to occur because of its relative short lifetime of $1.4 \mathrm{fs}$ [26]. Also, Table I shows that the transition dipole moments of the $Q_{2}{ }^{1} \Pi_{u}^{+}(1)$ and $Q_{1}{ }^{1} \Pi_{u}^{+}(1)$ doubly excited states only differ by a factor 2 [40]. Since the $Q_{1}{ }^{1} \Pi_{u}^{+}(1)$ and $Q_{2}{ }^{1} \Pi_{u}^{+}(1)$ doubly excited states located respectively at 34.2 and $36.3 \mathrm{eV}$ above the electronic ground state have comparable transition dipole moments, one expects QB-II effects between these doubly excited states with a period $2 \pi /\left(\epsilon_{k}-\epsilon_{k^{\prime}}\right) \sim 2$ fs to occur. Finally, if one retains only the contribution from the $Q_{2}{ }^{1} \Pi_{u}^{+}(1)$ doubly excited state by neglecting contributions from other doubly excited states, one can combine Eqs. (26) and (22) to predict that QB-I between the ground state and that doubly excited state is present in the fourth term (25) of the SDP (20) describing interference between the direct pathway $\Gamma_{2}^{\|}$and the indirect pathway $\Gamma_{12}^{\|}$. Below, we use TDSE calculations to demonstrate these three kinds of two-electron dynamics in the momentum, angular, and energy distributions.

\section{NUMERICAL RESULTS}

Our objective in presenting our numerical TDSE results is to show how molecular doubly excited state dynamics can affect the two-electron momentum, angular, and energy distributions in TPDI of the hydrogen molecule in the two schemes specified in Fig. 1, where the fixed-in-space diatomic molecule is aligned [Fig. 1(a)] or perpendicular [Fig. 1(b)] to the propagation direction of the laser beams. We only focus on time-delayed (nonoverlapping) oppositely circularly polarized attosecond pulses with a carrier frequency that is resonant with the transition between the field-free ground state and a specific doubly excited state. Thus, each cosine-squared pulse with $n=3$ cycles, zero CEP, and peak intensity $I=$ $50 \mathrm{TW} / \mathrm{cm}^{2}$ has a carrier frequency of $\omega \simeq 36 \mathrm{eV}$ for $\hat{\mathbf{k}} \| \hat{\mathbf{R}}$
[Fig. 1(a)] and of $\omega \simeq 30 \mathrm{eV}$ for $\hat{\mathbf{k}} \perp \hat{\mathbf{R}}$ [Fig. 1(b)]. These correspond to a pulse bandwidth $\Delta \omega \simeq 1.44 \omega / n$ of 17.3 and $14.4 \mathrm{eV}$, as well as to a pulse duration $T$ of 345 and 414 as. For each scheme we consider the orthogonal detection geometry and the in-plane back-to-back detection geometry in the case where the two electrons unequally share the excess energy $0.1 \leqslant E \precsim 50 \mathrm{eV}$ in the proportion $25 \%: 75 \%$. Below, while Secs. IV A and IV B are devoted to the investigation of doubly excited state effects on the momentum distributions for our two molecular orientations, Sec. IV C focuses on effects of doubly excited states on the angular distributions. Finally, Sec. IV D uses the $\varphi$-integrated energy distribution to isolate and demonstrate the autoionization decays and QB-II between doubly excited states.

For each of the momentum and angular distributions, we compare our complete TDSE results (i) with the TDSE results (ii) in which we exclude the ionization pathway corresponding to one-photon absorption from each pulse, specified by the pathway $\Gamma_{12}^{\|}$in Fig. 1(c) or the pathway $\Gamma_{12}^{\perp}$ in Fig. 1(d). For $\hat{\mathbf{k}} \| \hat{\mathbf{R}}$ in Fig. 1(a), this comparison is particularly dramatic for the two-electron momentum and angular distributions, whose fourfold rotational symmetry characters are completely changed by transitions corresponding to the indirect $\Gamma_{12}^{\|}$ pathway. In contrast, for $\hat{\mathbf{k}} \perp \hat{\mathbf{R}}$ in Fig. 1(b), this comparison reveals that the indirect $\Gamma_{12}^{\perp}$ pathway is necessary for the formation of four-arm spiral patterns in the momentum distributions.

\section{A. Effects of molecular doubly excited state on the momentum distribution for the pulse scheme $\hat{\mathbf{k}} \boldsymbol{\|} \mathbf{R}$}

For the case where the pair of nonoverlapping counterrotating circularly polarized attosecond pulses propagate along the fixed-in-space molecular axis $\mathbf{R}$, our TDSE results for the momentum $\mathbf{p}_{2}$ distribution for the in-plane BTB detection geometry are shown in Figs. 2(a) and 2(c), while those for the orthogonal detection geometry $\left(\mathbf{p}_{1} \perp \mathbf{p}_{2}\right)$ are shown in Figs. 2(b) and 2(d). For these two detection geometries, Fig. 2(a) and Fig. 2(b) show our TDSE results (ii) for the $\mathbf{p}_{2}$ distributions obtained numerically by including only the pathways $\Gamma_{1}^{\|}$and $\Gamma_{2}^{\|}$in the projection (1), i.e., they include final continuum states with $M= \pm 2$. Clearly one sees that the $\mathbf{p}_{2}$ distributions in the polarization plane for these two detection geometries exhibit regular spiral pattern with four arms. The handedness of the four-arm spirals observed in Figs. 2(a) and 2(b) for these two detection geometries is the same and dictated by the pulse helicities; it is counterclockwise for our time-delayed RCP/LCP pulses, and it changes to clockwise when switching the ordering of the two pulses (not shown). Moreover, the spiral pattern has a fourfold rotational symmetry since the four spiral arms are equally separated by $\pi / 2$. These TDSE results (ii) can be well understood using PT analysis. For these two detection geometries, it is very important that the dynamical amplitude $\mathcal{A}_{\Delta}\left(p_{1}, p_{2}, u, u_{1}, u_{2}\right)$ in the SDP (21) does not have any angular dependence since the mutual angles $u, u_{1}$, and $u_{2}$ are all kept fixed during the detection of the two photoelectrons. Indeed, $u=-1, u_{1}=$ $u_{2}=0$ in Fig. 2(a) for $\hat{\mathbf{p}}_{1}=-\hat{\mathbf{p}}_{2}$; while $u=u_{2}=0$, and $u_{1}=1$ for $\hat{\mathbf{p}}_{1} \perp \hat{\mathbf{p}}_{2}$. In the pulse polarization plane $(\theta=\pi / 2)$, 

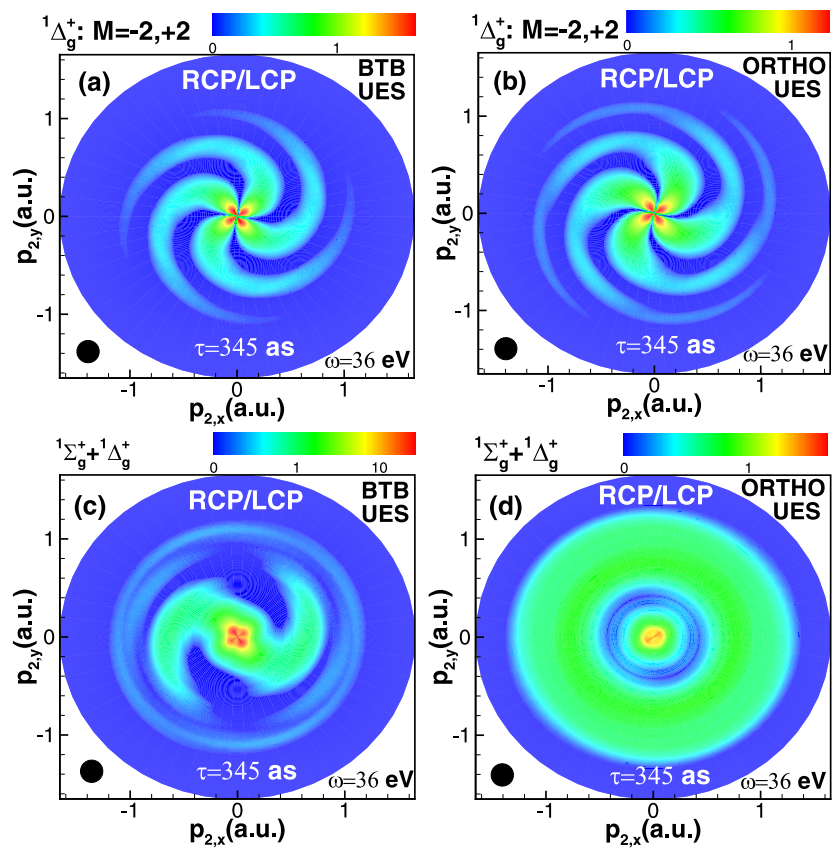

FIG. 2. Momentum $\mathbf{p}_{2}$ distributions [Eq. (1)] for TPDI of $\mathrm{H}_{2}$ by a pair of nonoverlapping, right-left, circularly polarized attosecond pulses $(\mathrm{RCP} / \mathrm{LCP})$ propagating along $\hat{\mathbf{k}}$ parallel to the molecular axis R. In the left panels [(a) and (c)], the two photoelectrons are emitted back-to-back (BTB) in the pulse polarization $x y$-plane. In the right panels [(b) and (d)], they are ejected orthogonally with one electron being fixed along $\hat{\mathbf{k}}$ and the second electron being recorded in the pulse polarization $x y$ plane. Top: Results when excluding the ionization ${ }^{1} \Sigma_{g}^{+}$channels. Bottom: Results when including the ionization channels ${ }^{1} \Sigma_{g}^{+}$and ${ }^{1} \Delta_{g}^{+}$. Each cosine-squared pulse in the pair has a carrier frequency $\omega \simeq 36 \mathrm{eV}$, peak intensity $I=50 \mathrm{TW} / \mathrm{cm}^{2}$, $\mathrm{CEP} \phi_{1}=\phi_{2}=0, n=3$ optical cycles, total duration $T \simeq 345$ as, and bandwidth $\Delta \omega \simeq 1.44 \omega / n=16.8 \mathrm{eV}$. The time delay between the two pulses is $\tau=T$. The two electrons unequally share (UES) the excess energy $0.1 \leqslant E \precsim 50 \mathrm{eV}$ in the proportion $25 \%: 75 \%$. In (a)-(d) the magnitudes of the SDP [in units of $10^{-6}$ a.u. (a), (c), and (d) and of $10^{-7}$ a.u. (b)] are indicated by the color scales. Here the polarization plane and the molecular plane are perpendicular.

kinematical vortex patterns are defined by the zeros and the maxima of the kinematical factor $\cos ^{2}(2 \xi \varphi-\Phi / 2)$ in the right-hand side of the SDP (21):

$$
\begin{aligned}
& \varphi^{\max }(E)=\xi\left[n \pi+\left(E-E_{g}\right) \tau+2 \phi_{12}\right] / 2, \\
& \varphi^{\text {zero }}(E)=\xi\left[n \pi+\pi / 2+\left(E-E_{g}\right) \tau+2 \phi_{12}\right] / 2,
\end{aligned}
$$

where $n=0, \pm 1, \pm 2, \ldots$ We note that Eq. (28) defines Archimedean spirals in the $(E, \varphi)$ plane. Given that $|\xi|=+1$, the number of spiral arms is four since $n$ can only take the values $0,1,2,3$; other values of $n$ will replicate the same lines. Also, these four arms of Archimedean spirals (28) are equally separated by $\pi / 2$, confirming the fourfold rotational symmetry seen in Figs. 2(a) and 2(b). We note that this kind of spiral patterns observed here for $\hat{\mathbf{k}} \| \hat{\mathbf{R}}$ were first predicted for single-photon [28] and multiphoton [29] attosecond single ionization of atoms with various and controlled number of spiral arms. They have now been confirmed experimentally [42-45] for femtosecond multiphoton ionization of $\mathrm{K}$ and $\mathrm{Na}$ atoms. Possible applications of kinematical electron vortices include laser pulse diagnostics with an emphasis as a pulse polarization photodetector, and control of electron motion on its natural attosecond timescale via the QB-III effect.

Figures 2(c) and 2(d) show the full TDSE results (i) for the $\mathbf{p}_{2}$ distributions in which the ionization pathway $\Gamma_{12}^{\|}$is now included in the projection (1), i.e., they include final continuum states with $M=0$ besides the $M= \pm 2$ ones. Note that the $Q_{2}{ }^{1} \Pi_{u}^{+}(1)$ doubly excited state and other doubly excited states with symmetry ${ }^{1} \Pi_{u}^{+}$are populated by one-photon transition from the first pulse thanks to its broad bandwidth $\Delta \omega=17.28 \mathrm{eV}$. One sees that the structure of the $\mathbf{p}_{2}$ distribution in the polarization plane changes dramatically, and it also strongly depends upon the detection geometry. For both detection geometries, one sees that the fourfold symmetry of the momentum distributions seen in Figs. 2(a) and 2(b) is destroyed by the contribution of the indirect ionization pathway $\Gamma_{12}^{\|}$. For the in-plane BTB detection geometry, the momentum distribution in Fig. 2(c) exhibits a two-arm spiral pattern for $p<1$ a.u.; together with a ringlike pattern for $p \simeq 1$ a.u. Despite the breakdown of the fourfold rotational symmetry of the spiral pattern as the time delay increases, we found that the two-arm spiral pattern for the in-plane BTB detection geometry is stable and its handedness is preserved. For the orthogonal detection geometry, the momentum distribution in Fig. 2(d) does not exhibit a spiral pattern, instead it exhibits a combination of dipolar patterns for momenta $p \preceq 0.3$ a.u. and circular symmetric patterns for momenta $p>0.7$ a.u., which are connected by dark and bright interferences fringe patterns for momenta $0.3<p<0.7$ a.u.

The difference in the shape of the $\mathbf{p}_{2}$ distributions observed in Figs. 2(c) and 2(d) for the two detection geometries can be understood using PT formula (20) by analyzing the relative magnitudes of its four terms and their angular structures. The relative magnitudes of these four terms are provided in Fig. 3, where we show the energy dependence of the dynamical parameters $\Gamma_{j}^{M}(E)$, with $M=0, \pm 2$ and $j=1,2,12$. Here $\Gamma_{1}^{+2}(E)=\left|\mathcal{A}_{\Delta}(\rho)\right|^{2}, \quad \Gamma_{2}^{-2}(E) \simeq\left|\mathcal{A}_{\Delta}(\rho)\right|^{2}, \quad$ and $\quad \Gamma_{12}^{0}(E) \equiv$ $\left|A_{\Gamma_{12}}(\rho, \theta ; \tau)\right|^{2}=\left|\mathcal{B}_{\Sigma}(\rho ; \tau) / 2+\mathcal{C}_{\Sigma}(\rho ; \tau)\right|^{2}$ describe the ionization pathways $\Gamma_{1}^{\|}, \Gamma_{2}^{\|}$, and $\Gamma_{12}^{\|}$, respectively. For $\tau=345$ as, these dynamical parameters are calculated numerically using our TDSE calculations by including in the projection (1) only final continuum states with $M=0$ for $j=12 ; M=+2$ for $j=1$; and $M=-2$ for $j=2$. Shown in Fig. 3(a) are results for the orthogonal geometry, while Fig. 3(b) shows results for the in-plane BTB geometry. One sees that the calculated parameters $\Gamma_{1}^{+2}(E)$ and $\Gamma_{2}^{-2}(E)$ are nearly identical for each of the two detection geometries, as correctly assumed by our PT. Over a wide excess energy range (except at very low excess energy $E$ ), the contrast between the calculated parameters $\Gamma_{12}^{0}(E)$ and $\Gamma_{1}^{+2}(E) \simeq \Gamma_{2}^{-2}(E)$ is much more larger for the orthogonal geometry [see Fig. 3(a)] than for the in-plane BTB geometry [see Fig. 3(b)]. According to PT formula (20) for the SDP, one sees that the last three terms of the SDP involves the ionization amplitude $A_{\Gamma_{12}}(\rho, \theta ; \tau)$ for the indirect ionization path $\Gamma_{12}^{\|}$. Thus, for the orthogonal geometry it is then clear that the shape of the $\mathbf{p}_{2}$ distribution in Fig. 2(d) over a wide excess energy range is dictated by the angular structure of the second term $\left|A_{\Gamma_{12}}(\rho, \theta ; \tau)\right|^{2}$ in the SDP (20), while for 

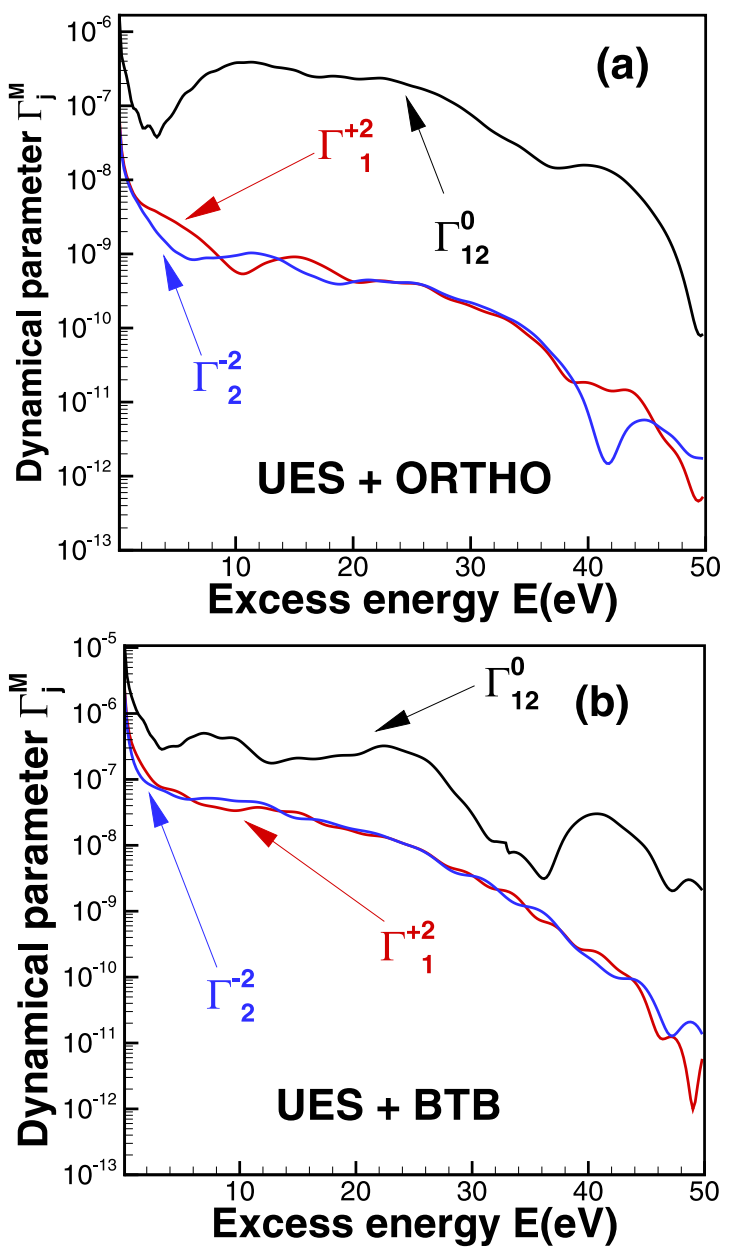

FIG. 3. Excess energy $E$ dependence on the dynamical parameters $\Gamma_{j}^{M}$ (where $j=1,2,12$ ) describing the ionization pathways $\Gamma_{1}^{\|}$, $\Gamma_{2}^{\|}$, and $\Gamma_{12}^{\|}$, respectively, in TPDI of $\mathrm{H}_{2}$ by a pair of nonoverlapping counter-rotating circularly polarized attosecond pulses propagating along the fixed-in-space molecular axis $\hat{\mathbf{k}} \| \mathbf{R}$. These parameters are extracted from TDSE calculations by including in the projection only final states with $M=0$ for $j=12 ; M=+2$ for $j=1$; and $M=-2$ for $j=2$. For an unequal energy sharing (UES) proportion of $25 \%: 75 \%$, two detection geometries are considered: (a) orthogonal geometry where $\mathbf{p}_{1} \| \hat{\mathbf{k}}$ and $\mathbf{p}_{2}$ detected in the laser polarization plane, and (b) back-to-back (BTB) emission of electrons in the laser polarization plane.

the in-plane BTB geometry the shape of the $\mathbf{p}_{2}$ distribution in Fig. 2(c) results from the strong interplay between the four terms in the SDP (20) given that these three dynamical parameters are comparable.

As the pump-probe term $\left|A_{\Gamma_{12}}(\rho, \theta ; \tau)\right|^{2}$ (23) is independent of the azimuthal angle $\varphi$ of the momentum unit vector $\hat{\mathbf{p}} \equiv \hat{\mathbf{p}}_{2}$, the breakdown of the fourfold rotational symmetry of the vortex pattern in Fig. 2(c) for the in-plane BTB geometry can only be due to the two interference terms (24) and (25), which differ in their kinematical structures. The kinematic factor $\cos ^{2}\left\{\left[E \tau+\phi_{12}+\Theta(\rho, \theta, \tau)\right] / 2-\xi \varphi\right\}$ of the interference term $2 \operatorname{Re}\left[A_{\Gamma_{1}}^{*} A_{\Gamma_{12}}\right]$ (24) leads to two-arm spiral vortex patterns with the same handedness as the four-arm spirals produced by the first term $\left|A_{\Gamma_{1}}+A_{\Gamma_{2}}\right|^{2}(21)$ of the SDP. How- ever, the spiral equations for this two-arm spiral,

$$
\begin{aligned}
& \varphi^{\max }(E)=-\xi\left[n \pi-\left(E \tau+\phi_{12}+\Theta\right) / 2\right], \\
& \varphi^{\text {zero }}(E)=-\xi\left[n \pi+\pi / 2-\left(E \tau+\phi_{12}+\Theta\right) / 2\right],
\end{aligned}
$$

are modified by the dynamical phase $\Theta(\rho, \theta ; \tau)(26)$. Correspondingly, the dipolelike kinematic factor $\sin ^{2}\left\{\left[E_{g} \tau-\phi_{12}+\right.\right.$ $\Theta(\rho, \theta, \tau)] / 2+\xi \varphi\}$ of the interference term $2 \operatorname{Re}\left[A_{\Gamma_{2}}^{*} A_{\Gamma_{12}}\right]$ (25) cannot support a spiral vortex structure in the momentum distribution since its dependence on the excess energy $E$ is only via the dynamical phase $\Theta(\rho, \theta ; \tau)(26)$. We then conclude that the change of shape from the fourfold to twofold rotational symmetry of the vortex patterns occurring at $p<1$ a.u. when comparing Figs. 2(a) and 2(c) for the in-plane BTB geometry originates only from the interference term $2 \operatorname{Re}\left[A_{\Gamma_{1}}^{*} A_{\Gamma_{12}}\right]$ (24). In contrast, for $p>1$ a.u. for which $\Gamma_{12}^{0}(E)$ is much greater than $\Gamma_{1}^{+2}(E) \simeq \Gamma_{2}^{-2}(E)$, the change of shape from four-arm spiral pattern to a ringlike pattern observed when comparing Figs. 2(a) and 2(c) is an indication of the dominance of the isotropic pump-probe term $\left|A_{\Gamma_{12}}(\rho, \theta ; \tau)\right|^{2}(23)$ in the SDP (20). Such dominance of the isotropic pump-probe term seen in Fig. 3(a) for the orthogonal detection geometry for $p>0.7$ a.u. leads to a circularly symmetric pattern in Fig. 2(d) that obscures the occurrence of any two-arm spiral pattern. For $p<0.7$ a.u., this isotropic pump-probe term seen in Fig. 3(a) becomes less dominant such that the shape of the $\mathbf{p}_{2}$ distribution in Fig. 2(d) is now controlled by the interplay between the last three terms in the SDP (20) that leads to a dipolelike pattern exhibiting some dark and bright interference fringes between the two regions.

All the results for the momentum distributions, calculated using Eqs. (1) and (2), presented in Fig. 2 and analyzed by Fig. 3 are obtained within the fixed-nuclei approximation at the equilibrium internuclear distance $R \simeq 1.4$ a.u. To freeze any nuclear motion between the electron transitions for the TPDI process involving doubly excited state channels, we have used sufficiently short pulses to make the molecular TPDI problem more atomiclike; and also used time delays $\tau$ shorter than $\tau_{d}$, which depends on the isotope, see the second paragraph of Sec. III. For each $\tau$, stable vortex patterns such as the one in Fig. 2(c) are found. However, there is unavoidably the vibrational broadening due to the quantum uncertainty principle (zero-point energy) and nuclear motion in the final state that are always present and thus need to be investigated. When considering nuclear motion in the initial state, the small zero-point energy $\omega_{0} / 2=\sqrt{k_{m} / 4 \mu}$ of $0.27 \mathrm{eV}$ for $\mathrm{H}_{2}, 0.19 \mathrm{eV}$ for $\mathrm{D}_{2}$, and $0.15 \mathrm{eV}$ for $\mathrm{T}_{2}$ raises the energy of the ground state above the minimum of the $U(R)$ curve. As the field-free $Q_{2}{ }^{1} \Pi_{u}^{+}(1)$ doubly excited state is located at $36.3 \mathrm{eV}$ above this minimum (see Table I), the corresponding resonant carrier frequency $\omega$ is thus $36.03 \mathrm{eV}$ for $\mathrm{H}_{2}$, $36.11 \mathrm{eV}$ for $\mathrm{D}_{2}$, and $36.15 \mathrm{eV}$ for $\mathrm{T}_{2}$. In order to calculate the TPDI probability within the adiabatic-nuclei approximation using Eq. (9), TDSE calculations of $\mathcal{W}\left(\mathbf{p}_{1}, \mathbf{p}_{2} ; R_{c}\right)$ for nine values of the classical turning point $R_{c}$ are carried out within the Franck-Condon region. For each isotope, all the TDSE calculations for different $R_{c}$ are done for the same carrier frequency, durations, and time delays of three cycles. Using the initial-state vibrational wave function $\chi_{0}\left(R_{c}\right)$ displayed in Fig. 4(a) and defined by Eq. (4) within the harmonic- 

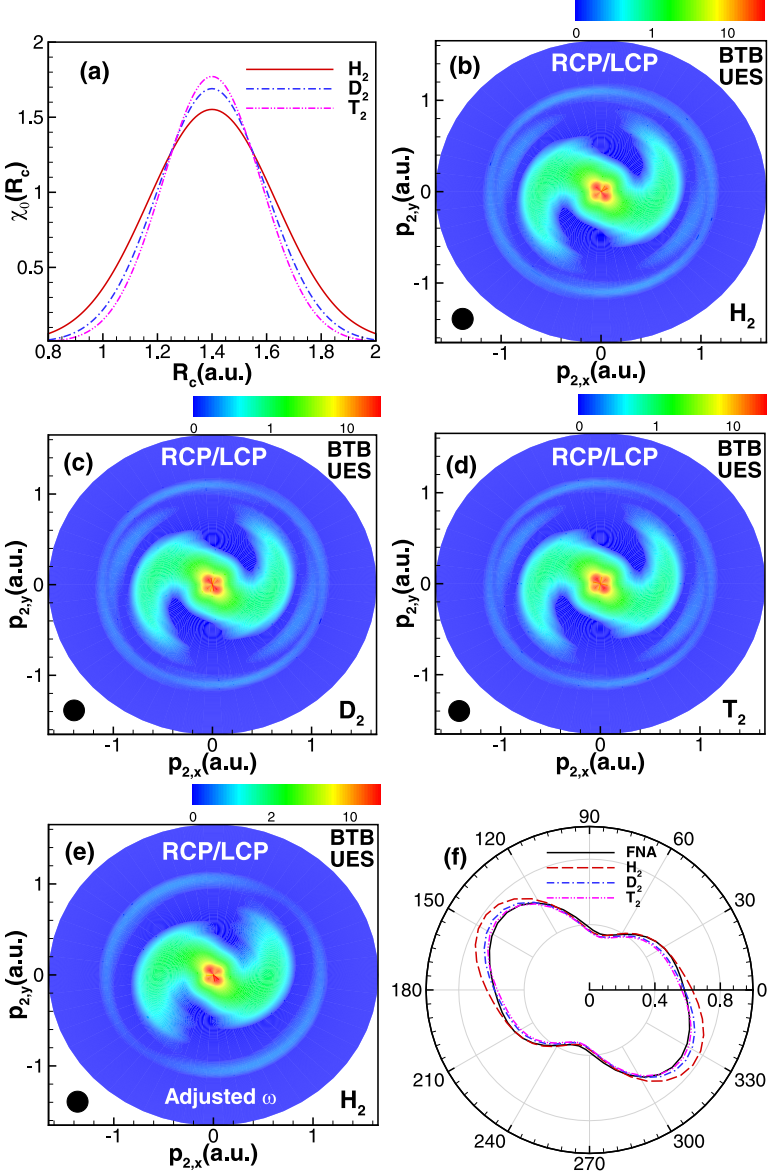

FIG. 4. (a) Initial-state vibrational wave function $\chi_{0}\left(R_{c}\right)$ given by Eq. (4) within the harmonic-oscillator approximation. (b)-(d) The $\mathbf{p}_{2}$ distributions calculated using Eq. (9) within the adiabatic-nuclei approximation for TPDI of (b) $\mathrm{H}_{2}$, (c) $\mathrm{D}_{2}$, and (d) $\mathrm{T}_{2}$. The small zero-point energy $\omega_{0} / 2=\sqrt{k_{m} / 4 \mu}$ of $0.27 \mathrm{eV}$ for $\mathrm{H}_{2}, 0.19 \mathrm{eV}$ for $\mathrm{D}_{2}$, and $0.15 \mathrm{eV}$ for $\mathrm{T}_{2}$ raises the energy of the ground state above the minimum of the $U(R)$ curve. As the field-free $Q_{2}{ }^{1} \Pi_{u}^{+}(1)$ doubly excited state is located at $36.3 \mathrm{eV}$ above this minimum (see Table I), the corresponding resonant carrier frequency $\omega$ is $36.03 \mathrm{eV}$ for $\mathrm{H}_{2}, 36.11 \mathrm{eV}$ for $\mathrm{D}_{2}$, and $36.15 \mathrm{eV}$ for $\mathrm{T}_{2}$. TDSE calculations of $\mathcal{W}\left(\mathbf{p}_{1}, \mathbf{p}_{2} ; R_{c}\right)$ for a given isotope at different values of $R_{c}$ are done for the same carrier frequency, pulse duration, and time delay of three cycles. (e) The same as in (b), but calculated by adapting the carrier frequency $\omega$ such that the same electronic state is reached for different values of $R_{c}$. In (b)-(e), the magnitudes of the SDP (in units of $10^{-6}$ a.u.) are indicated by the color scales. (f) In units of $10^{-6}$ a.u. the energy-integrated angular distribution in which the result extracted from Fig. 2(c) for the fixed-nuclei approximation (FNA) is compared with the three isotopic results (b)-(d) for the adiabaticnuclei approximation. The two electrons are emitted back-to-back (BTB) in the pulse polarization $x y$ plane and unequally share (UES) the excess energy $0.1 \leqslant E \precsim 50 \mathrm{eV}$ in the proportion $25 \%: 75 \%$. Other pulse parameters are specified in the caption of Fig. 2.

oscillator approximation, our TDSE results for the momentum distributions calculated using Eq. (9) are shown in Fig. 4(b) for $\mathrm{H}_{2}$, Fig. 4(c) for $\mathrm{D}_{2}$, and Fig. 4(d) for $\mathrm{T}_{2}$. For a given isotope one sees that the zero-point energy due to nuclear motion does not erase the vortex pattern signature, since the patterns in Figs. 4(b)-4(d) greatly resemble the one displayed in Fig. 2(c). Indeed, Figs. 4(b)-4(d) and Fig. 2(c) have the same shape and only differ slightly in magnitudes, which can be explained by the little difference seen in Fig. 4(a) for the Gaussian-shaped vibrational wave function $\chi_{0}\left(R_{c}\right)$ for these three isotopes. The same conclusion about the persistence of vortex patterns from these three isotopes is obtained when calculating the momentum distribution using Eq. (9) by adapting the pulse carrier frequency $\omega$ such that the same intermediate electronic state is reached for different values of $R_{c}$, as illustrated in Fig. 4(e) for $\mathrm{H}_{2}$.

Another observable relevant to probe the applicability of fixed-nuclei approximation with respect to adiabatic-nuclei approximation is the energy-integrated angular distribution shown in Fig. 4(f). Plotted in this figure are the result extracted from Fig. 2(c) within the fixed-nuclei approximation and the three isotopic results extracted from Figs. 4(b)-4(d) within the adiabatic-nuclei approximation. While Fig. 4(f) shows that the angular distributions for all these isotopes have the same shape, the difference in magnitudes induced by nuclei vibrations is rather little. Very striking is the observation in Fig. 4(a) that $\chi_{0}(R)$-already peaked about the equilibrium internuclear distance $R_{e}$ for $\mathrm{H}_{2}$ - becomes strongly peaked about $R_{e}$ when the reduced mass $\mu$ of the isotope increases. Consequently, according to the discussion below Eq. (9) the validity of the fixed-nuclei approximation should be more effective for heavier isotopes. Our numerical results in Fig. 4(f) confirm those expectations. The contrast between results for the adiabatic-nuclei and fixed-nuclei approximations is largest for the lightest isotope $\mathrm{H}_{2}$, and smallest for the heaviest isotope $\mathrm{T}_{2}$. As $\mu$ increases (from $\mathrm{H}_{2}$ to $\mathrm{T}_{2}$ via $\mathrm{D}_{2}$ ) the angular distributions obtained within the adiabatic-nuclei approximation converge towards the angular distribution obtained within the fixed-nuclei approximation. Although the treatment of this resonant TPDI process within the adiabatic-nuclei approximation is more realistic and accurate, one sees that its simplistic treatment within the fixed-nuclei approximation provides a very good account.

\section{B. Effects of molecular doubly excited state on the momentum distribution for the pulse scheme $\hat{\mathbf{k}} \perp \mathbf{R}$}

For the case where the pair of nonoverlapping counterrotating circularly polarized attosecond pulses propagate perpendicularly to the fixed-in-space molecular axis $\mathbf{R}$, we only consider the orthogonal detection geometry in which one electron is emitted along the pulse propagation direction $\mathbf{p}_{1} \| \hat{\mathbf{k}}$ and the other is emitted in the polarization $x y$ plane, as illustrated in Fig. 1(b). We do not consider the in-plane BTB detection geometry since it is known [16] that the larger contrast between the ${ }^{1} \Pi_{u}^{+}$and ${ }^{1} \Sigma_{u}^{+}$ionization amplitudes in such detection scheme does not favor the observation of spiral patterns in the momentum distribution when purely circularly polarized attosecond pulses delayed in time are used to double photoionize the hydrogen molecule in the configuration $\hat{\mathbf{k}} \perp \mathbf{R}$.

Our TDSE results for the $\mathbf{p}_{2}$ distribution for the orthogonal detection geometry is shown in Fig. 5(a) when only the two direct ionization channels $\Gamma_{1}^{\perp}$ and $\Gamma_{2}^{\perp}$ in Fig. 1(b) are included in the TDSE calculations. In other words, here the contri- 

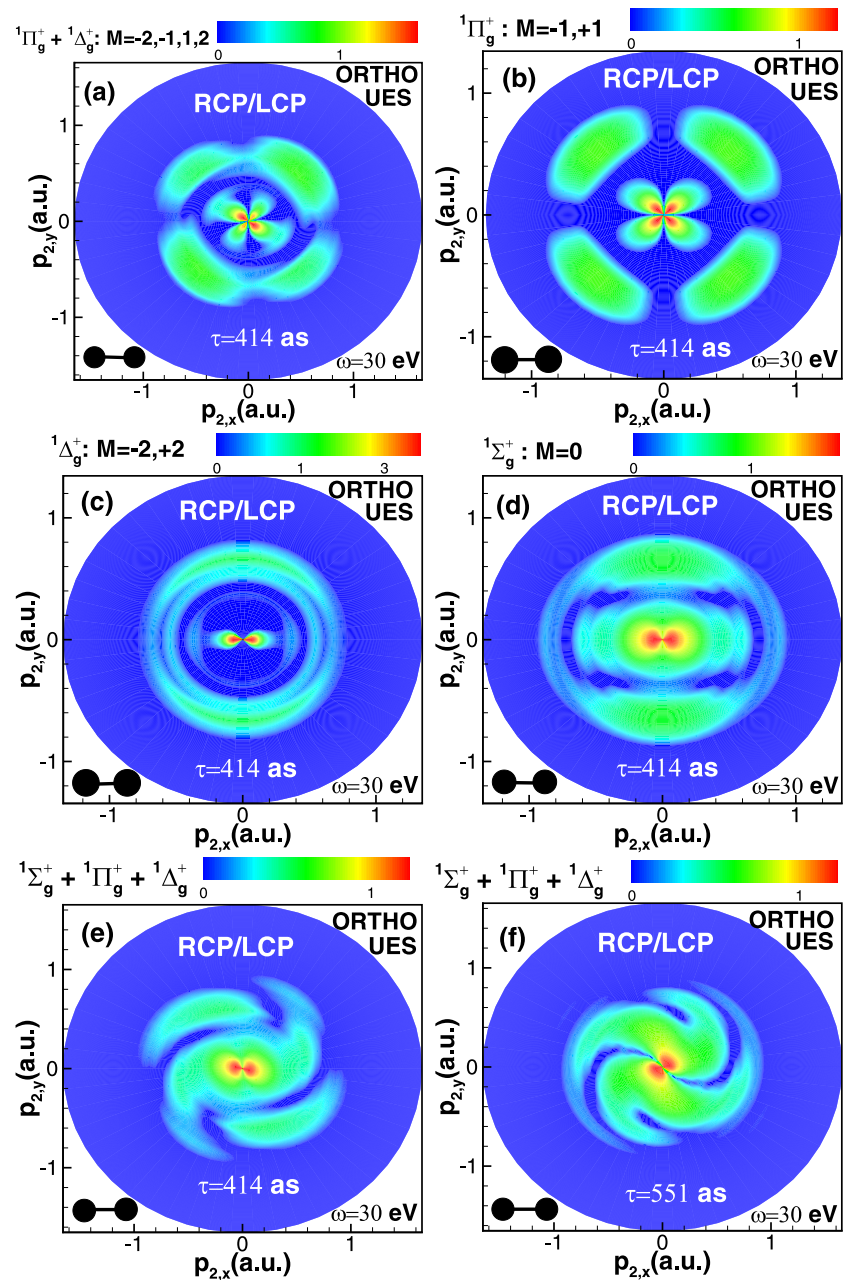

FIG. 5. Momentum $\mathbf{p}_{2}$ distributions [Eq. (1)] for TPDI of $\mathrm{H}_{2}$ by a pair of nonoverlapping, right-left, circularly polarized attosecond pulses (RCP/LCP) delayed in time by $\tau=414$ as and propagating along $\hat{\mathbf{k}}$ perpendicular to the molecular axis $\mathbf{R}$. The $\mathbf{p}_{2}$ distributions are obtained by TDSE calculations that include: (a) the ${ }^{1} \Pi_{g}^{+}$and ${ }^{1} \Delta_{g}^{+}$continuum states with $M= \pm 2, \pm 1$; (b) the ${ }^{1} \Pi_{g}^{+}$continuum states with $M= \pm 1$; (c) the ${ }^{1} \Delta_{g}^{+}$continuum states with $M= \pm 2$; (d) the ${ }^{1} \Sigma_{g}^{+}$continuum states with $M=0$; and (e) the ${ }^{1} \Sigma_{g}^{+},{ }^{1} \Pi_{g}^{+}$, and ${ }^{1} \Delta_{g}^{+}$continuum states with $M=0, \pm 1, \pm 2$. (f) The same as (e) but for a time delay $\tau=551$ as, i.e., one cycle longer. The two photoelectrons unequally share (UES) the excess energy $0.1 \leqslant E \precsim$ $50 \mathrm{eV}$ in the proportion $25 \%: 75 \%$; they are ejected orthogonally with one electron being fixed along $\hat{\mathbf{k}}$ and the second electron being recorded in the pulse polarization $x y$ plane. The pulse parameters are: a carrier frequency $\omega=30 \mathrm{eV}$, peak intensity $I=50 \mathrm{TW} / \mathrm{cm}^{2}, \mathrm{CEP}$ $\phi_{1}=\phi_{2}=0$, optical cycles $n=3$, total duration $T \simeq 414$ as, and bandwidth $\Delta \omega \simeq 1.44 \omega / n=14.4 \mathrm{eV}$. The magnitudes of the SDP [in units of $10^{-6}$ a.u. (a), (b), and (d), $10^{-7}$ a.u. (c), and $10^{-5}$ a.u. (e) and (f)] are indicated by the color scales. Here the polarization plane coincides with the molecular plane.

butions for the indirect ionization channel $\Gamma_{12}^{\perp}$ in Fig. 1(b) are omitted. In contrast to the case of pulse configuration $\hat{\mathbf{k}} \| \mathbf{R}$, one sees that this momentum distribution obtained for $\hat{\mathbf{k}} \perp \mathbf{R}$ does not present any helical structure. Instead, it does exhibit two sets of twofold symmetric quadrupole patterns well separated in energy, with one appearing at lower energies delimitated by $p<0.6$ a.u. and the other appearing at higher energies delimitated by $p>0.6$ a.u. In each of these two sets, the two spots along the direction $\varphi \sim \pi / 4,5 \pi / 4$ with respect to the molecular $x$ axis have the same intensity. This is also the case for the two spots along the direction $\varphi \sim 3 \pi / 4,7 \pi / 4$. However, for the low-energy set the pair of spots along the direction $\varphi \sim \pi / 4,5 \pi / 4$ are less brighter than those along the direction $\varphi \sim 3 \pi / 4,7 \pi / 4$; while for the high-energy set the pair of spots along the direction $\varphi \sim \pi / 4,5 \pi / 4$ are more brighter than those along the direction $\varphi \sim 3 \pi / 4,7 \pi / 4$.

To understand the twofold symmetric quadrupolelike shape of the $\mathbf{p}_{2}$ distribution shown in Fig. 5(a), we note that according to the electric dipole selection rules the first two-electron wave packet is created through the direct ionization pathway $\Gamma_{1}^{\perp}$, which describes the transitions ${ }^{1} \Sigma_{g}^{+} \rightarrow\left[{ }^{1} \Pi_{u}^{+}(M=\right.$ $\left.+1),{ }^{1} \Sigma_{u}^{+}(M=0)\right] \rightarrow\left[{ }^{1} \Delta_{g}^{+}(M=+2),{ }^{1} \Pi_{g}^{+}(M=+1)\right]$ for two-photon absorption by the RCP pulse from the ground state to the double continua. In the following, $A_{\Pi}^{(1)}$ and $A_{\Delta}^{(1)}$ are used to denote the ionization amplitudes corresponding respectively to these two final continuum states. Likewise, the second two-electron wave packet is created through the direct ionization pathway $\Gamma_{2}^{\perp}$, which describes the transitions ${ }^{1} \Sigma_{g}^{+} \rightarrow\left[{ }^{1} \Pi_{u}^{+}(M=-1),{ }^{1} \Sigma_{u}^{+}(M=0)\right] \rightarrow\left[{ }^{1} \Delta_{g}^{+}(M=\right.$ $\left.-2),{ }^{1} \Pi_{g}^{+}(M=-1)\right]$ for two-photon absorption by the LCP pulse from essentially the same ground state to the double continua. We also use $A_{\Pi}^{(2)}$ and $A_{\Delta}^{(2)}$ to denote the ionization amplitudes corresponding respectively to these two final continuum states. Since the two oppositely circularly polarized attosecond pulses are delayed in time by $\tau$, the second twoelectron wave packet is created when the first two-electron wave packet has already accumulated a Ramsey phase $\Phi$ given by Eq. (17). Therefore, the momentum distribution in Fig. 5(a) is obtained by superposing three kinds of patterns:

$$
\begin{aligned}
\mathcal{W}_{\Gamma_{1}^{\perp}}^{\Gamma_{2}^{\perp}}= & \left|\left[A_{\Pi}^{(1)}+A_{\Delta}^{(1)}\right]+e^{i \Phi}\left[A_{\Pi}^{(2)}+A_{\Delta}^{(2)}\right]\right|^{2} \\
= & \left|\left[A_{\Pi}^{(1)}+e^{i \Phi} A_{\Pi}^{(2)}\right]+\left[A_{\Delta}^{(1)}+e^{i \Phi} A_{\Delta}^{(2)}\right]\right|^{2} \\
= & \left|A_{\Pi}^{(1)}+e^{i \Phi} A_{\Pi}^{(2)}\right|^{2}+\left|A_{\Delta}^{(1)}+e^{i \Phi} A_{\Delta}^{(2)}\right|^{2} \\
& +2 \operatorname{Re}\left[\left(A_{\Pi}^{(1) *}+e^{-i \Phi} A_{\Pi}^{(2) *}\right)\left(A_{\Delta}^{(1)}+e^{i \Phi} A_{\Delta}^{(2)}\right)\right] .
\end{aligned}
$$

In the SDP (30), the first term $\left|A_{\Pi}^{(1)}+e^{i \Phi} A_{\Pi}^{(2)}\right|^{2}$ describes Ramsey interference between the ${ }^{1} \Pi_{g}^{+}(M= \pm 1)$ components of the created pair of two-electron wave packets; the second term $\left|A_{\Delta}^{(1)}+e^{i \Phi} A_{\Delta}^{(2)}\right|^{2}$ describes Ramsey interference between the ${ }^{1} \Delta_{g}^{+}(M= \pm 2)$ components of the created pair of the two-electron wave packets; and the third term $2 \operatorname{Re}\left[\left(A_{\Pi}^{(1) *}+e^{-i \Phi} A_{\Pi}^{(2) *}\right)\left(A_{\Delta}^{(1)}+e^{i \Phi} A_{\Delta}^{(2)}\right)\right]$ describes the mixing of the ${ }^{1} \Pi_{g}^{+}(M= \pm 1)$ and ${ }^{1} \Delta_{g}^{+}(M= \pm 2)$ ionization amplitudes within each two-electron wave packet or in between the created pair of two-electron wave packets. Figure 5(b) on the one hand shows that Ramsey interference from the first term $\left|A_{\Pi}^{(1)}+e^{i \Phi} A_{\Pi}^{(2)}\right|^{2}$ in the SDP (30) leads to two sets of fourfold symmetric quadrupolelike patterns with the four spots in each of the two sets having the same intensity. Figure 5(c) on the other hand shows that Ramsey interference from the second term $\left|A_{\Delta}^{(1)}+e^{i \Phi} A_{\Delta}^{(2)}\right|^{2}$ in the SDP (30) leads to two sets of patterns with the one at lower energies being a 
figure-eight-shaped dipole along the molecular $x$ axis and the other at higher energies being a broadside oval-shaped dipole along the $y$ direction perpendicular to the molecular $x$ axis. However, the magnitude of the SDP in Fig. 5(c) is one order of magnitude smaller than the SDPs in Figs. 5(a) and 5(b). Therefore, the four spots seen in Fig. 5(a) indicate that the leading channels in the $\mathbf{p}_{2}$ distribution stems from the ionization paths $\Gamma_{1}^{\perp}$ with the ${ }^{1} \Pi_{g}^{+}(M=+1)$ final continuum state and $\Gamma_{2}^{\perp}$ with the ${ }^{1} \Pi_{g}^{+}(M=-1)$ final continuum state. Moreover, all these TDSE results in Figs. 5(b) and 5(c) show that the difference in the brightness of the quadrupolelike pattern seen in Fig. 5(a) is thus due to the third term $2 \operatorname{Re}\left[\left(A_{\Pi}^{(1) *}+\right.\right.$ $\left.\left.e^{-i \Phi} A_{\Pi}^{(2) *}\right)\left(A_{\Delta}^{(1)}+e^{i \Phi} A_{\Delta}^{(2)}\right)\right]$ in the SDP (30), which mixes the ionization amplitudes produced either from the same path or from different path, but with different molecular symmetry.

In Fig. 5(e) we show the $\mathbf{p}_{2}$ distribution for the orthogonal detection geometry and the pulse configuration $\hat{\mathbf{k}} \perp$ $\mathbf{R}$ when the two direct ionization channels $\Gamma_{1}^{\perp}$ and $\Gamma_{2}^{\perp}$ in Fig. 1(b), as well as the indirect ionization pathway $\Gamma_{12}^{\perp}$, are all included in the TDSE calculations. Owing to the broad pulse bandwidth $\Delta \omega \simeq 1.44 \omega / n=14.4 \mathrm{eV}$, the pulse carrier frequency of $\omega=30 \mathrm{eV}$ is such that $Q_{1}{ }^{1} \Pi_{u}^{+}(1)$, $Q_{1}{ }^{1} \Sigma_{u}^{+}(1), Q_{2}{ }^{1} \Pi_{u}^{+}(1)$, and $Q_{1}{ }^{1} \Sigma_{u}^{+}(2)$ doubly excited states are all populated by one-photon absorption from the first pulse. These molecular doubly excited states are thus involved in the indirect ionization pathway $\Gamma_{12}^{\perp}$ in Fig. 1(b). Therefore, this indirect ionization pathway $\Gamma_{12}^{\perp}$ describes the transitions ${ }^{1} \Sigma_{g}^{+} \rightarrow\left[{ }^{1} \Pi_{u}^{+}(M=+1),{ }^{1} \Sigma_{u}^{+}(M=0)\right] \rightarrow\left[{ }^{1} \Sigma_{g}^{+}(M=\right.$ $\left.0),{ }^{1} \Pi_{g}^{+}(M=-1)\right]$ for one-photon absorption by the RCP pulse from the ground state to doubly excited states, followed by one-photon absorption by the LCP pulse from the populated doubly excited states to the double continua. Figure 5(d) shows that the momentum distribution, obtained by projecting the two-electron wave packet onto only final ${ }^{1} \Sigma_{g}^{+}(M=0)$ continuum state, exhibits two sets of broadside oval-shaped dipolelike patterns along and perpendicular to the molecular $x$ axis. We note that the magnitude of the SDPs in Figs. 5(a) and 5(d) are comparable. Consequently, thanks to strong interference the $\mathbf{p}_{2}$ distribution in Fig. 5(e) is just a superposition of the twofold symmetric quadrupolelike pattern in Fig. 5(a) with the two sets of broadside oval-shaped dipolelike patterns in Fig. 5(d).

Surprisingly, one sees that the $\mathbf{p}_{2}$ distribution in Fig. 5(e) produced by time-delayed right-left circularly polarized attosecond pulses presents a counterclockwise spiral pattern with four arms. Inverting the ordering of the two pulses leads to a clockwise spiral pattern (not shown). Moreover, increasing the time delay $\tau$ between the pulses results in tightly wound spiral patterns, as illustrated in Fig. 5(f) when $\tau$ in Fig. $5(\mathrm{e})$ is increased by one cycle, $T_{0}=2 \pi / \omega \simeq 138$ as. Although the ionization pathway $\Gamma_{12}^{\perp}$ involves the final continuum state ${ }^{1} \Pi_{g}^{+}(M=-1)$, the comparison between Figs. 5(a) and 5(e) probes qualitatively the contribution of the indirect ionization pathway $\Gamma_{12}^{\perp}$-involving doubly excited statesrelative to the direct ionization pathways $\Gamma_{1}^{\perp}$ and $\Gamma_{2}^{\perp}$. We can conclude that just as a strong interplay between the ${ }^{1} \Pi_{u}^{+}(M=$ $+1)$ and ${ }^{1} \Sigma_{u}^{+}(M=0)$ continuum amplitudes produced in single-photon double ionization of $\mathrm{H}_{2}$ was found to lead to dynamical electron vortices with two spiral arms [16], here the dynamical electron vortices with four spiral arms seen in Figs. 5(e) and 5(f) stems from a strong overlap between the leading ${ }^{1} \Pi_{g}^{+}(M= \pm 1)$ and ${ }^{1} \Sigma_{g}^{+}(M=0)$ ionization amplitudes. The difference in the number of spiral arms is related to the number of absorbed photons in a given process. While one photon was absorbed from each pulse in DPI of $\mathrm{H}_{2}$ [16], here two photons are absorbed from each pulse for our resonant TPDI of $\mathrm{H}_{2}$ within the $\hat{\mathbf{k}} \perp \hat{\mathbf{R}}$ configuration.

\section{Effects of molecular doubly excited states on the angular distributions at fixed excess energy}

In this section, for only the pulse configuration $\hat{\mathbf{k}} \| \hat{\mathbf{R}}$, we consider the energy-sharing configuration and resonant pulse parameters used in Sec. IV A to study whether the angular distributions for a fixed energy $E=2 \omega+E_{g}$ exhibit QB-I between the ${ }^{1} \Sigma_{g}^{+}$ground state and the $Q_{2}{ }^{1} \Pi_{u}^{+}(1)$ doubly excited state. Our focus is on the in-plane BTB detection geometry for which the indirect ionization pathway $\Gamma_{12}^{\|}$involving doubly excited states is demonstrated above in Sec. IV A to change the character of the vortex pattern from four-arm spiral pattern to two-arm spiral pattern thanks to its strong interference with the two direct paths $\Gamma_{1}^{\|}$and $\Gamma_{2}^{\|}$. As the SDP in Sec. IV A for the orthogonal detection geometry is essentially controlled by the indirect ionization pathway $\Gamma_{12}^{\|}$, this case is not considered because there is almost no interference.

For the in-plane BTB detection geometry where $\omega \simeq 36.14$ $\mathrm{eV}$, we show in Fig. 6 the angular distributions in the pulse polarization $x y$ plane for a fixed excess energy $E=2 \omega+E_{g}$ and five time delays: $\tau=T=343$ as [Fig. 6(a)]; $\tau=T+$ $T_{0} / 4 \simeq 372$ as [Fig. 6(b)]; $\tau=T+T_{0} / 2 \simeq 401$ as [Fig. 6(c)]; $\tau=T+3 T_{0} / 4 \simeq 429$ as [Fig. 6(d)]; and $\tau=T+T_{0} \simeq 458$ as [Fig. 6(e)], where $T_{0}=2 \pi / \omega \simeq 114$ as is the pulse optical period. In each panel of Fig. 6 we compare our TDSE results (i) obtained by projecting the two-electron wave packet onto the ${ }^{1} \Delta_{g}^{+}(M= \pm 2)$ and ${ }^{1} \Sigma_{g}^{+}(M=0)$ final continuum states with our TDSE results (ii) for the ${ }^{1} \Delta_{g}^{+}(M= \pm 2)$ final continuum states. For each time delay $\tau$ in Fig. 6, comparing the angular distributions (i) and (ii) probes both qualitatively and quantitatively the contribution of the indirect ionization pathway $\Gamma_{12}^{\|}$-involving the $Q_{2}{ }^{1} \Pi_{u}^{+}(1)$ doubly excited state and other ${ }^{1} \Pi_{u}^{+}$doubly excited states [e.g., $\left.Q_{1}{ }^{1} \Pi_{u}^{+}(1)\right]$ listed in Table I-relative to the direct ionization pathways $\Gamma_{1}^{\|}$and $\Gamma_{2}^{\|}$. The angular distribution (ii) in Fig. 6 at any time delay $\tau$ exhibits a fourfold symmetric quadrupolelike shape. In contrast, the shape of the angular distribution (i) in Fig. 6 varies with the time delay $\tau$, changing from a twofold symmetric quadrupole shape to a broadside oval-dipole shape or even to a peanutdipole shape. As $\tau$ increases, the angular distributions (ii) rotate counterclockwise with an angular shift $\Delta \varphi$ specified in Table II. Meanwhile, the angular distributions (i) also rotate in the same direction and at the same frequency. From Fig. 6 and Table II one sees that the angular distributions (ii) do have a periodicity with increasing time delay $\tau$ of $\pi / \omega=T_{0} / 2 \simeq 57$ as; while the angular distributions (i) in Fig. 6 do not.

Let us explain all these TDSE results (i) and (ii) using PT. First, the angular distributions (ii) resulting from Ramsey interference between the created pair of two-electron wave packets produced by the two direct ionization pathways $\Gamma_{1}^{\|}$ 


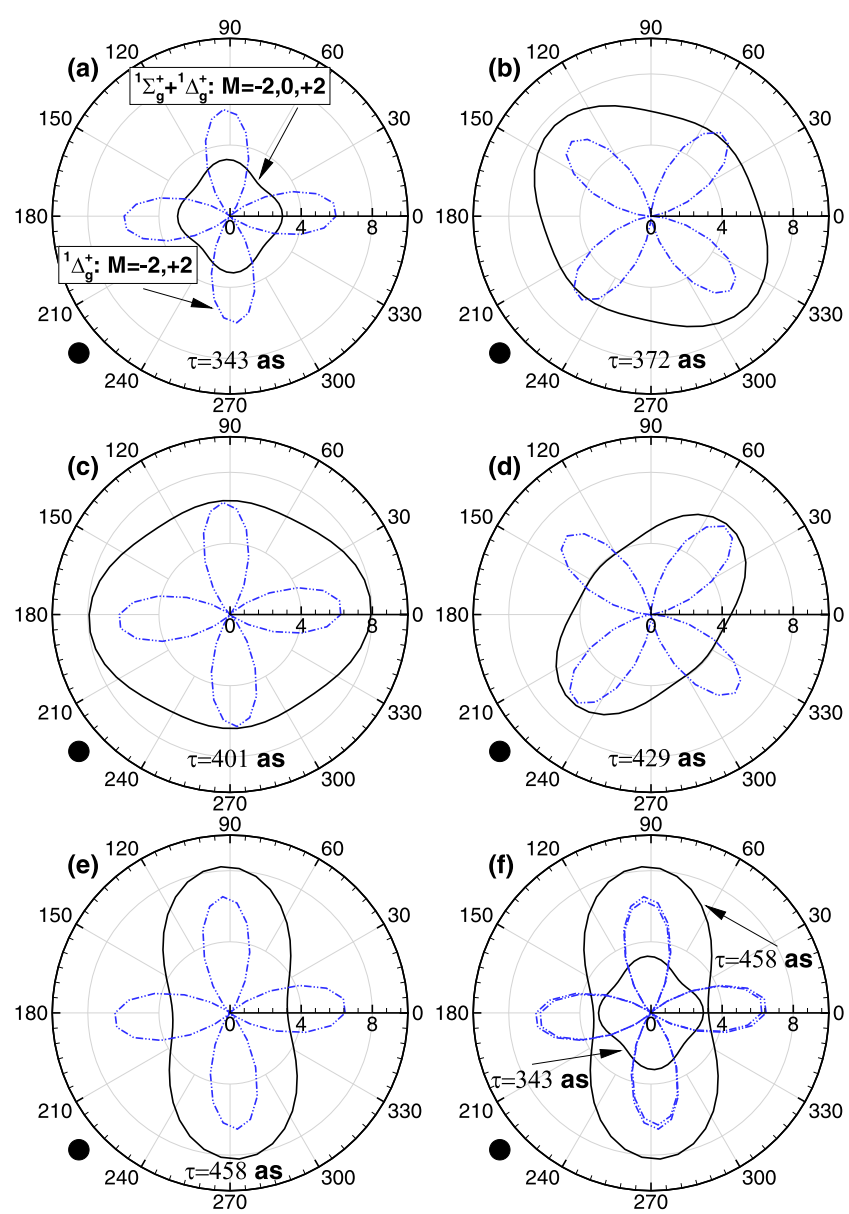

FIG. 6. Angular distributions (in units of $10^{-7}$ a.u.) in TPDI of the hydrogen molecule initially in the ground state at $R=1.4$ a.u. for a fixed excess energy $E=2 \omega-E_{b} \simeq 20.9 \mathrm{eV}$ produced by a pair of nonoverlapping, right-left, circularly polarized attosecond pulses having a relative CEP $\phi_{12}=0$, an intensity $I=50 \mathrm{TW} / \mathrm{cm}^{2}$ for five values of the time delay $\tau$ : (a) $\tau=T \simeq 343$ as; (b) $\tau=T+T_{0} / 4 \simeq$ 372 as; (c) $\tau=T+T_{0} / 2 \simeq 401$ as; (d) $\tau=T+3 T_{0} / 4 \simeq 429$ as; and (e) $\tau=T+T_{0} \simeq 458$ as, where $T_{0}=2 \pi / \omega$. The pulse carrier frequency $\omega=36.14 \mathrm{eV}$ is resonant with the $Q_{2}{ }^{1} \Pi_{u}^{+}(1)$ doubly excited state; however, the broad pulse bandwidth $\Delta \omega=17.28 \mathrm{eV}$ is such that other doubly excited states [e.g., $\left.Q_{1}{ }^{1} \Pi_{u}^{+}(1)\right]$ are also involved. The solid (black) curves are our TDSE results (i) including all paths $\Gamma_{1}^{\|}, \Gamma_{2}^{\|}$, and $\Gamma_{12}^{\|}$, which lead to final states ${ }^{1} \Sigma_{g}^{+}(M=0)$ and ${ }^{1} \Delta_{g}^{+}(M= \pm 2)$. The dash double-dotted (blue) curves are our TDSE results (ii) scaled by a factor 10 including only the paths $\Gamma_{1}^{\|}$ and $\Gamma_{2}^{\|}$, which lead to final states ${ }^{1} \Delta_{g}^{+}(M= \pm 2)$. In panel (f), we compare results in (a) and (e) to show the presence and absence of $\tau$ periodicity in results (ii) and (i), respectively. The degree of energy sharing is fixed to $25 \%: 75 \%$, and electrons are emitted back-to-back in the laser polarization plane.

and $\Gamma_{2}^{\|}$is described by PT formula (21). For a fixed excess energy $E=2 \omega+E_{g}, \Phi=\left(E-E_{g}\right) \tau+2 \phi_{12}=2\left(\omega \tau+\phi_{12}\right)$ such that the angular distribution (ii) given by (21) becomes

$$
\mathcal{W}_{\Gamma_{1}^{\|}}^{\Gamma_{2}^{\|}}=\left|\mathcal{A}_{\Delta}(\rho)\right|^{2} \cos ^{2}\left(\omega \tau+\phi_{12}-2 \xi \varphi\right)
$$

It is clear from its $\varphi$ dependence that the angular distribution (31) exhibits a fourfold symmetric quadrupolelike shape.
TABLE II. Angular shift $\Delta \varphi(\tau)$ of the angular distributions as a function of the time-delay $\tau$ at the fixed excess energy $E=20.926$ $\mathrm{eV}$ in Fig. 6 with respect to the horizontal axis.

\begin{tabular}{cccccc}
\hline \hline$\tau$ (as) & 343 & 372 & 401 & 429 & 458 \\
$\Delta \varphi(\tau)$ & $0^{\circ}$ & $45^{\circ}$ & $90^{\circ}$ & $135^{\circ}$ & $180^{\circ}$ \\
\hline \hline
\end{tabular}

Also, it has a periodicity with increasing time delay $\tau$ of $\pi / \omega$. These predictions for the shape and periodicity are well consistent with our TDSE results (ii) reported in Fig. 6 and Table II.

Second, the shape of the angular distribution (i) is described by PT formula (20) with its four terms given by PT formulas (31), (23), (24), and (25). For a fixed time delay $\tau$, since the second term (23) in (20) does not depend upon the azimuthal angle $\varphi$ of the momentum $\mathbf{p}_{2}$, the $\varphi$ dependence of the ionization probability is thus controlled by the interplay between the first term (31) in (20)_-discussed above-with the two interference terms (24) and (25). Indeed, for a fixed excess energy $E=2 \omega+E_{g}$ and in the pulse polarization plane $(\theta=\pi / 2)$, the first interference term (24) becomes

$$
\begin{aligned}
2 \operatorname{Re} & {\left[A_{\Gamma_{1}}^{*} A_{\Gamma_{12}}\right] \propto\left|\mathcal{A}_{\Delta}^{*}(\rho) A_{\Gamma_{12}}(\rho, \theta, \tau)\right| } \\
& \times \cos ^{2}\left\{\phi_{12} / 2+\omega \tau+\left[E_{g} \tau+\Theta(\rho, \theta, \tau)\right] / 2-\xi \varphi\right\},
\end{aligned}
$$

while the second interference term (25) becomes

$$
\begin{aligned}
2 \operatorname{Re} & {\left[A_{\Gamma_{2}}^{*} A_{\Gamma_{12}}\right] \propto-\left|\mathcal{A}_{\Delta}^{*}(\rho) A_{\Gamma_{12}}(\rho, \theta, \tau)\right| } \\
& \times \sin ^{2}\left\{\phi_{12} / 2-\left[E_{g} \tau+\Theta(\rho, \theta, \tau)\right] / 2-\xi \varphi\right\} .
\end{aligned}
$$

From their $\varphi$ dependence, one sees that the two interference terms (32) and (33) present a dipolar shape; however, their periodicity with increasing time delay $\tau$ are different and strongly depend on the interplay of the molecular doubly excited states via the dynamical phase $\Theta(\rho, \theta, \tau)$ (26). Indeed, if $\Theta(\rho, \theta, \tau)(26)$ were to be linear in $\tau$ with a coefficient $\tilde{\Theta}$, then the period for the interference term (32) would be $2 \pi /\left[2 \omega+\left(E_{g}+\tilde{\Theta}\right)\right]$ while that for the interference term (33) would be $2 \pi /\left|E_{g}+\tilde{\Theta}\right|$. Our TDSE results in Fig. 3(b) for the in-plane BTB detection geometry show that the magnitude of the dynamical parameter $\left|\mathcal{A}_{\Delta}(\rho)\right|^{2}$, which is proportional to the transition probabilities for the paths $\Gamma_{1}^{\|}$and $\Gamma_{2}^{\|}$, is comparable to the magnitude of the dynamical parameter $\left|\mathcal{A}_{\Delta}^{*}(\rho) A_{\Gamma_{12}}(\rho, \theta, \tau)\right|$ in the interference terms involving either the path $\Gamma_{1}^{\|}$or the path $\Gamma_{2}^{\|}$with the path $\Gamma_{12}^{\|}$. Therefore, the effects of constructive and destructive interference of the three $\varphi$-dependent terms in (20) become clear. The interplay between the first term $\left|A_{\Gamma_{1}}+A_{\Gamma_{2}}\right|^{2}$ (31) and the interference term $2 \operatorname{Re}\left[A_{\Gamma_{1}}^{*} A_{\Gamma_{12}}\right]$ (32) produces two large lobes in the angular distribution (i) in one direction, whereas the interplay between this first term (31) and the interference term $2 \operatorname{Re}\left[A_{\Gamma_{2}}^{*} A_{\Gamma_{12}}\right]$ (33) produces two small lobes in the angular distribution (i) in a perpendicular direction. These positive and negative interference effects lead to a twofold symmetric quadrupolelike shape for the angular distribution (i) observed in Fig. 6.

Although several $Q$ doubly excited states with ${ }^{1} \Pi_{u}^{+}$symmetry are populated by one-photon transition thanks to the 
broad pulse bandwidth, the $Q_{2}{ }^{1} \Pi_{u}^{+}(1)$ doubly excited state with energy $\epsilon_{Q_{2}{ }^{1} \Pi_{u}^{+}(1)}$ has the largest oscillator strength among those states. Retaining only the contribution from this state in the derivation of the dynamical phase $\Theta(\rho, \theta, \tau)(26)$, one gets

$$
\Theta(\rho, \theta, \tau) \simeq \alpha_{Q_{2}{ }^{1} \Pi_{u}^{+}(1)}(\rho, \theta)-\epsilon_{Q_{2}{ }^{1} \Pi_{u}^{+}(1)} \tau,
$$

where $\alpha_{Q_{2}{ }^{1} \Pi_{u}^{+}(1)}(\rho, \theta)$ is the phase of the dynamical parameter $\mathcal{A}_{\Delta}^{*}(\rho) A_{\Gamma_{12}}^{Q_{2}{ }^{1} \Pi_{u}^{+}(1)}$ describing the interference of the path $\Gamma_{1}^{\|}$or $\Gamma_{2}^{\|}$with the path $\Gamma_{12}^{\|}$. In this case, one reads from Eq. (34) that $\tilde{\Theta}=-\epsilon_{Q_{2}{ }^{1} \Pi_{u}^{+}(1)}$ leads to the same period of $T_{0}=2 \pi / \omega \simeq 114$ as for both interference terms (32) and (33), where $\omega \simeq\left(\epsilon_{Q_{2}{ }^{1} \Pi_{u}^{+}(1)}-E_{g}\right)$ is the beat frequency between the ${ }^{1} \Sigma_{g}^{+}$ground state and the $Q_{2}{ }^{1} \Pi_{u}^{+}(1)$ doubly excited state. Moreover, as the time delay $\tau$ increases, the resulting twofold symmetric quadrupolelike shape of the angular distribution (i) should thus rotate counterclockwise with a frequency $\omega$ and should remain unchanged, i.e., be periodic when $\tau=n(2 \pi / \omega)$, where $n$ is an integer. While this prediction for the rotation direction for the angular distribution (i) as $\tau$ varies is quite satisfied in Fig. 6, the differences in both the shape and the magnitude of the angular distributions (i) at different values of $\tau$ evidence the absence of its time-delay periodicity. In other words, the QB-I between the ${ }^{1} \Sigma_{g}^{+}$ground state and the $Q_{2}{ }^{1} \Pi_{u}^{+}(1)$ doubly excited state has an anomaly in shape and magnitude, but not in frequency. These effects are clearly visible in Fig. 6(f) where the angular distributions (i) for $\tau=343$ as and $\tau=343+T_{0}=458$ as have the same orientation, but they do not coincide.

The fact that the angular distributions (i) rotate with increasing $\tau$ at the correct QB-I frequency $\omega \simeq\left(\epsilon_{Q_{2}{ }^{1} \Pi_{u}^{+}(1)}-E_{g}\right)$ between the ${ }^{1} \Sigma_{g}^{+}$ground state and the $Q_{2}{ }^{1} \Pi_{u}^{+}(1)$ doubly excited state is a strong indication that the approximation (34) may be used. In other words, the interplay between that doubly excited state and other $Q$ doubly excited states with ${ }^{1} \Pi_{u}^{+}$symmetry (listed in Table I) involved in the dynamical phase $\Theta(\rho, \theta, \tau)$ (26) does not affect this QB-I frequency. Furthermore, the reported anomaly in shape and magnitude of this QB-I effect is a clear indication that besides the $Q_{2}{ }^{1} \Pi_{u}^{+}(1)$ doubly excited state, other $Q$ doubly excited states with ${ }^{1} \Pi_{u}^{+}$symmetry may significantly contribute to this resonant TPDI process either through the QB-II process or autoionization decays, which is the goal of the next subsection. Indeed, the $Q_{2}{ }^{1} \Pi_{u}^{+}(1)$ doubly excited state has a relatively short lifetime of 1.4 fs [26], while its transition dipole moment is only a factor 2 larger than that for the $Q_{1}{ }^{1} \Pi_{u}^{+}(1)$ doubly excited state [40]. Therefore, for attosecond time delays used here the QB-II phenomenon between the $Q_{1}{ }^{1} \Pi_{u}^{+}(1)$ and $Q_{2}{ }^{1} \Pi_{u}^{+}(1)$ doubly excited states at a frequency $\left(\epsilon_{Q_{1}{ }^{1} \Pi_{u}^{+}(1)}-\epsilon_{Q_{2}{ }^{1} \Pi_{u}^{+}(1)}\right)$ together with autoionization decays may significantly change the magnitude of the dynamical parameter $\left|\mathcal{A}_{\Delta}^{*}(\rho) A_{\Gamma_{12}}(\rho, \theta, \tau)\right|$ (27), which enters in both the interference terms (32) and (33). These two time-dependent processes may also change the magnitude of the pump-probe term $\left|A_{\Gamma_{12}}(\rho, \theta, \tau)\right|^{2}$ (23). To show how the two-electron dynamics of autoionization and QB-II effects are responsible for the anomaly in the QB-I effect between the ground state and $Q_{2}{ }^{1} \Pi_{u}^{+}(1)$ doubly excited state, below we use PT to analyze the TDSE results for the time delay sensitivity of the energy distributions obtained by integrating the SDPs over the azimuthal angle $\varphi$ of the photoelectron pair.

\section{Dynamics of autoionization and QB-II between doubly} excited states revealed in the $\varphi$-integrated energy distributions

To remove any QB-I phenomenon in the energy distributions but remain sensitive to the two-electron dynamics of autoionization decays and QB-II between doubly excited states, we integrate the SDP (20) over the azimuthal angle $\varphi$ of the electron pair and obtain

$$
\mathcal{W}(\rho, \theta, \tau)=\pi \sin ^{4}(\theta)\left|\mathcal{A}_{\Delta}(\rho)\right|^{2}+2 \pi\left|A_{\Gamma_{12}}(\rho, \theta, \tau)\right|^{2} .
$$

The $\varphi$-integrated energy distribution (35) is comprised of only the first two terms of the SDP (20), as the interference terms (24) and (25) between the path $\Gamma_{12}^{\|}$(involving among others the QB-I effect) and either $\Gamma_{1}^{\|}$or $\Gamma_{2}^{\|}$vanish upon integrating over $\varphi$. As the first term in Eq. (35) is independent of the time delay $\tau$, its energy distribution $\propto\left|\mathcal{A}_{\Delta}(\rho)\right|^{2}$ in the polarization plane $(\theta=\pi / 2)$ has the shape shown in Fig. 3. Strikingly, its second term depends on the time delay $\tau$. From Eq. (23), that second term $\propto\left|A_{\Gamma_{12}}(\rho, \theta, \tau)\right|^{2}$ involves autoionization decays and QB-II effects. Therefore, the $\varphi$-integrated energy distribution (35) appears to be a unique observable for exploring these two kinds of two-electron dynamics. For the same detection scheme and pulse parameters used in Fig. 2(c), our TDSE results for the $\varphi$-integrated energy distribution (i) in the polarization plane $(\theta=\pi / 2)$ are shown in Fig. 7 for four values of the time delay considered in Fig. 6. Clearly Fig. 7(a) on a log scale shows that the $\varphi$-integrated energy distribution (i) is very sensitive to the time delay $\tau$, which is due to the combined effects of autoionization decays and QB-II according to PT formula (35). Also, these TDSE results are independent of the relative CEP $\phi_{12}$, a result which is consistent with the PT result (35). Finally, the vertical dashed line shown in Fig. 7(b) on a linear scale marks the excess energy at which the angular distributions in Fig. 6 are presented. As the time delay $\tau$ between the two pulses varies, a careful analysis of the relative magnitude of the probability at that excess energy permits to fully understand the anomaly in shape and magnitude of the QB-I effect seen in Fig. 6 for the $Q_{2}{ }^{1} \Pi_{u}^{+}(1)$ doubly excited state.

\section{SUMMARY AND CONCLUSIONS}

In summary, using both analytical tools and numerical TDSE toolboxes we have investigated the correlated, resonant process of TPDI of the hydrogen molecule via doubly excited states by using time-delayed and nonoverlapping oppositely circularly polarized attosecond pulses. Sufficiently short attosecond pulses and time delays are used to make the molecular problem more atomiclike by freezing nuclei motion in the intermediate states. We have treated this problem within the adiabatic-nuclei approximation (including nuclei vibrational motion in both the initial and final states) or within the fixed-nuclei approximation, and found that the latter is valid as it provides a very good account for this process. 

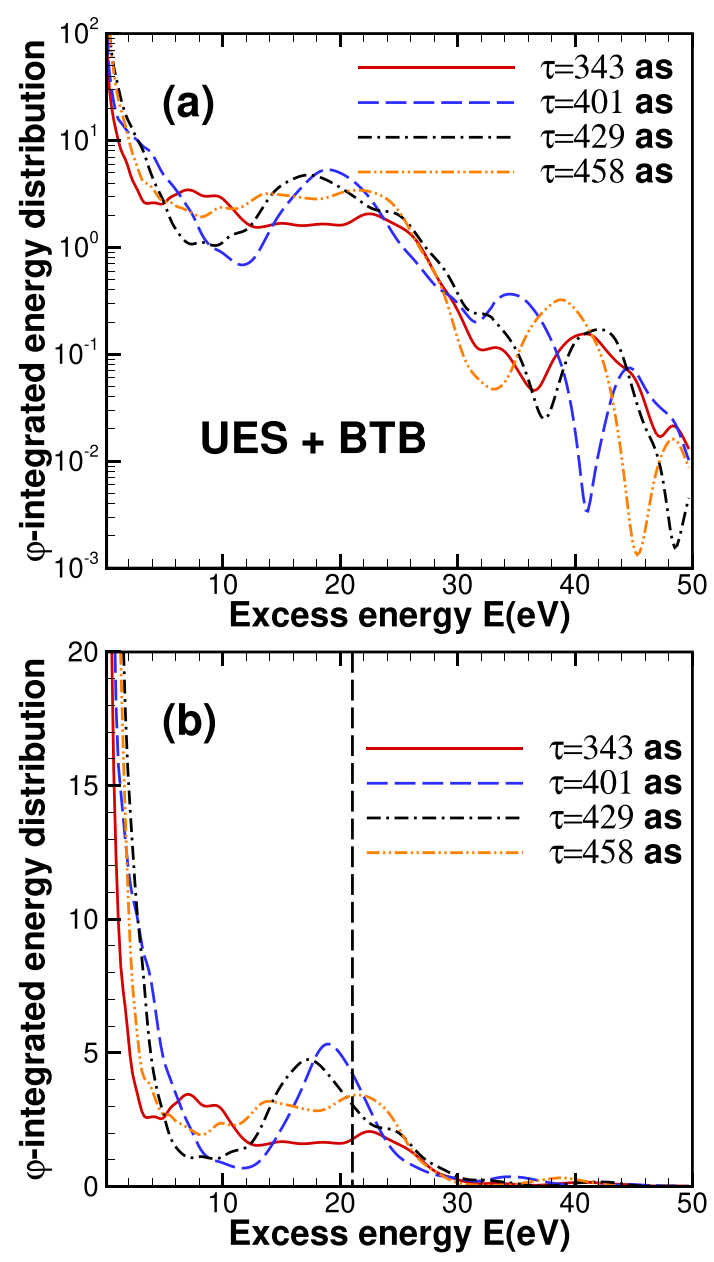

FIG. 7. TDSE results (in units of $10^{-6}$ a.u.) on a log scale (a) or linear scale (b) for the energy distributions obtained by integrating the SDP (1) over the azimuthal angle $\varphi$ of the photoelectron pair. Results are shown for four values of the time delay $\tau$ considered in Fig. 6 and analyzed using PT formula (35). The two electrons are detected back-to-back (BTB) in the laser polarization plane $(\theta=\pi / 2)$ and unequally share (UES) the excess energy $0.1 \leqslant E \precsim 50 \mathrm{eV}$ in the proportion $0.25: 0.75$. The vertical dashed line in (b) marks the position of the energy $E=2 \omega-E_{b} \simeq 20.9 \mathrm{eV}$. The other parameters are specified in the caption for Fig. 2(c).

For the pulse direction $\hat{\mathbf{k}}$ either parallel or perpendicular to the fixed-in-space molecular axis $\hat{\mathbf{R}}$, we have shown that exquisite temporal coherent control for this resonant process can be achieved by interfering three ionization pathways. These include the two direct ionization pathways and the indirect ionization pathway that involves several doubly excited states, which are populated by the first circularly polarized pulse. For the two photoelectrons unequally sharing the excess energy, we have considered two detection geometries for which the mutual angle $\beta=\cos ^{-1}\left(\hat{\mathbf{p}}_{1} \cdot \hat{\mathbf{p}}_{2}\right)$ between the two electron momenta is always kept constant. Our study revealed that this resonant process of TPDI of the hydrogen molecule is very sensitive to the molecular orientation with respect to the laser pulse direction. Different patterns appear in the two-electron momentum distribution in the polarization plane when $\hat{\mathbf{k}} \| \hat{\mathbf{R}}$ or $\hat{\mathbf{k}} \perp \hat{\mathbf{R}}$, since ionization amplitudes with different molecular symmetry are excited via the three pathways according to the electric dipole selection rules and they are mixed.

In the former case $(\hat{\mathbf{k}} \| \hat{\mathbf{R}})$, just as for He atoms [14], the indirect ionization path is found to change the character of the fourfold rotational symmetry of the momentum distributions produced by Ramsey interference of the two direct ionization paths; however, in contrast of atoms the angular distributions here for molecules present a QB-I effect [between the ground state and the strongest $Q_{2}{ }^{1} \Pi_{u}^{+}(1)$ doubly excited state] with an anomaly in shape and magnitude, not in frequency. QBII between doubly excited states together with autoionization decays are shown to be mainly responsible for the reported QB-I anomaly.

In the latter case $(\hat{\mathbf{k}} \perp \hat{\mathbf{R}})$, the momentum distribution resulting from the superposition of these three ionization paths exhibits counterintuitively a pattern of four-arm spiral dynamical vortices, which reflects not only the number of photons absorbed per pulse from the ground state and their handedness, but also the relative magnitude and phase of the interfering leading ${ }^{1} \Pi_{g}^{+}(M= \pm 1)$ and ${ }^{1} \Sigma_{g}^{+}(M=0)$ ionization amplitudes. For both the parallel and perpendicular molecular orientations, we found that the vortex spiral patterns remain stable upon inclusion of vibrational broadening due to the quantum uncertainty relation (zero-point energy).

We have only considered the cases of the purely parallel and perpendicular molecular orientations. To get a complete picture one must consider the case of an arbitrarily oriented $\mathrm{H}_{2}$ molecule. However, such a numerical project is beyond the scope of the present study where the so-called $M$-mixing problem due to elliptically polarized pulses must be solved for each molecular direction. For future works, we note from Ref. [46] for a linearly polarized laser light that the isotropic rotational distribution for DPI of randomly oriented hydrogen molecules obtained by integrating the SDP over the molecular orientation $\hat{\mathbf{R}}$ is just a sum of the parallel contribution weighted by $1 / 3$ and perpendicular contribution weighted by $2 / 3$. For TPDI, it is already more complicated as this observable involves not only terms arising from the purely parallel and perpendicular orientation, but also a number of mixed and interference terms.

The experimental observation of our predictions for the different patterns present in the momentum distributions for both parallel and perpendicular molecular orientations requires the broad bandwidth of isolated nonoverlapping attosecond pulses. Indeed, the momentum distributions for the strictly parallel and perpendicular configurations can be post-selected (within a certain aperture) in recoil-ion and cold target recoilion momentum spectroscopy (COLTRIMS) experiments [47]. Evolving from COLTRIMS, reaction microscope techniques [48] already exist for measuring TPDI momentum distributions. Also, electron vortices [28,29] with full control of the number of spiral arms have been demonstrated experimentally in multiphoton ionization of potassium or sodium using either single-color or two-color femtosecond time-delayed laser pulses [42-45] using velocity map imaging (VMI). Finally, 
isolated attosecond pulses with controlled polarization have been realized [49].

\section{ACKNOWLEDGMENTS}

This work was supported by the US Department of Energy (DOE), Office of Science, Basic Energy Sciences (BES), un- der Award No. DE-FG03-96ER14646 (J.M.N.D. and A.F.S.) and Award No. DE-SC0021054 (J.M.N.D.). Computations were carried out using Stampede 2 at Texas Advanced Computing Center (TACC) under Grant No. TG-PHY-120003. This work was completed utilizing the Holland Computing Center of the University of Nebraska, which receives support from the Nebraska Research Initiative. J.M.N.D. thanks I. Fabrikant for helpful discussions on reflection principles.
[1] P. Brumer and M. Shapiro, Control of unimolecular reactions using coherent light, Chem. Phys. Lett. 126, 541 (1986).

[2] S. Shi, A. Woody, and H. Rabitz, Optimal control of selective vibrational excitation in harmonic linear chain molecules, J. Chem. Phys. 88, 6870 (1988).

[3] R. Kosloff et al., Wavepacket dancing: Achieving chemical selectivity by shaping light pulses, Chem. Phys. 139, 201 (1989).

[4] A. H. Zewail, Femtochemistry: Ultrafast Dynamics of the Chemical Bond (World Scientific, Singapore, 1994).

[5] V. Blanchet, C. Nicole, M.-A. Bouchene, and B. Girard, Temporal Coherent Control of Two-Photon Transitions: From Optical Interferences to Quantum Interferences, Phys. Rev. Lett. 78, 2716 (1997).

[6] D. B. Foote, Y. Lin, L.-W. Pi, J. M. Ngoko Djiokap, A. F. Starace, and W. T. Hill, III, Ionization enhancement and suppression by phase-locked ultrafast pulse pairs, Phys. Rev. A 96, 023425 (2017).

[7] N. F. Ramsey, A molecular beam resonance method with separated oscillating fields, Phys. Rev. 78, 695 (1950).

[8] P. Tzallas, E. Skantzakis, L. A. A. Nikolopoulos, G. D. Tsakiris, and D. Charalambidis, Extreme-ultraviolet pump-probe studies of one-femtosecond-scale electron dynamics, Nat. Phys. 7, 781 (2011).

[9] G. Sansone, L. Poletto, and M. Nisoli, High-energy attosecond light sources, Nat. Photon. 5, 655 (2011).

[10] S. X. Hu, and L. A. Collins, Attosecond Pump Probe: Exploring Ultrafast Electron Motion Inside an Atom, Phys. Rev. Lett. 96, 073004 (2006).

[11] T. Morishita, S. Watanabe, and C. D. Lin, Attosecond Light Pulses for Probing Two-Electron Dynamics of Helium in the Time Domain, Phys. Rev. Lett. 98, 083003 (2007).

[12] J. Feist, S. Nagele, C. Ticknor, B. I. Schneider, L. A. Collins and J. Burgdörfer, Attosecond Two-Photon Interferometry for Doubly Excited States of Helium, Phys. Rev. Lett. 107, 093005 (2011).

[13] W.-C. Jiang, W.-H. Xiong, T.-S. Zhu, L.-Y. Peng, and Q. Gong, Double ionization of $\mathrm{He}$ by time-delayed attosecond pulses, J. Phys. B 47, 091001 (2014).

[14] J. M. N. Djiokap and A. F. Starace, Doubly excited state effects on two-photon double ionization of helium by timedelayed, oppositely circularly-polarized attosecond pulses, J. Opt. 19, 124003 (2017).

[15] C.-N. Liu, M.-K. Chen, and C. D. Lin, Radiative decay of helium doubly excited states, Phys. Rev. A 64, 010501(R) (2001).

[16] J. M. Ngoko Djiokap, A. V. Meremianin, N. L. Manakov, L. B. Madsen, S. X. Hu, and A. F. Starace, Dynamical electron vortices in attosecond double photoionization of $\mathrm{H}_{2}$, Phys. Rev. A 98, 063407 (2018).
[17] J. M. Ngoko Djiokap, A. V. Meremianin, N. L. Manakov, S. X. $\mathrm{Hu}, \mathrm{L}$. B. Madsen, and A. F. Starace, Kinematical vortices in double photoionization of helium by attosecond pulses, Phys. Rev. A 96, 013405 (2017).

[18] I. A. Ivanov and A. S. Kheifets, Time-dependent calculations of double photoionization of the aligned $\mathrm{H}_{2}$ molecule, Phys. Rev. A 85, 013406 (2012).

[19] X. Guan, K. Bartschat, and B. I. Schneider, Breakup of the aligned $\mathrm{H}_{2}$ molecule by XUV laser pulses: A time-dependent treatment in prolate spheroidal coordinates, Phys. Rev. A 83, 043403 (2011).

[20] W. Vanroose, F. Martín, T. N. Rescigno, and C. W. McCurdy, Complete photo-induced breakup of the $\mathrm{H}_{2}$ molecule as a probe of molecular electron correlation, Science 310, 1787 (2005).

[21] W. Vanroose, D. A. Horner, F. Martín, T. N. Rescigno, and C. W. McCurdy, Double photoionization of aligned molecular hydrogen, Phys. Rev. A 74, 052702 (2006).

[22] J. Colgan, M. S. Pindzola, and F. Robicheaux, Triple Differential Cross Sections for the Double Photoionization of $\mathrm{H}_{2}$, Phys. Rev. Lett. 98, 153001 (2007).

[23] L. Tao, C. W. McCurdy, and T. N. Rescigno, Grid-based methods for diatomic quantum scattering problems. III. Double photoionization of molecular hydrogen in prolate spheroidal coordinates, Phys. Rev. A 82, 023423 (2010).

[24] W.-C. Jiang, L.-Y. Peng, J.-W. Geng, and Q. Gong, One-photon double ionization of $\mathrm{H}_{2}$ with arbitrary orientation, Phys. Rev. A 88, 063408 (2013).

[25] J. M. Ngoko Djiokap, A. V. Meremianin, N. L. Manakov, L. B. Madsen, S. X. Hu, and A. F. Starace, Molecular SymmetryMixed Dirchroism in Double Photoionization of $\mathrm{H}_{2}$, Phys. Rev. Lett. 123, 143202 (2019).

[26] J. Tennyson, Resonance parameters and quantum defects for superexcited $\mathrm{H}_{2}$, At. Data. Nucl. Data Tables 64, 253 (1996).

[27] J. M. Ngoko Djiokap, N. L. Manakov, A. V. Meremianin, S. X. $\mathrm{Hu}, \mathrm{L}$. B. Madsen, and A. F. Starace, Nonlinear Dichroism in Back-to-Back Double Ionization of He by an Intense Elliptically Polarized Few-Cycle Extreme Ultraviolet Pulse, Phys. Rev. Lett. 113, 223002 (2014).

[28] J. M. Ngoko Djiokap, S. X. Hu, L. B. Madsen, N. L. Manakov, A. V. Meremianin, and A. F. Starace, Electron Vortices in Photoionization by Circularly Polarized Attosecond Pulses, Phys. Rev. Lett. 115, 113004 (2015).

[29] J. M. Ngoko Djiokap, A. V. Meremianin, N. L. Manakov, S. X. $\mathrm{Hu}, \mathrm{L}$. B. Madsen, and A. F. Starace, Multistart spiral electron vortices in ionization by circularly polarized pulses, Phys. Rev. A 94, 013408 (2016). 
[30] C. W. McCurdy, M. Baertschy, and T. N. Rescigno, Solving the three-body Coulomb breakup problem using exterior complex scaling, J. Phys. B 37, R137 (2004).

[31] S. X. Hu, Optimizing the FEDVR-TDCC code for exploring the quantum dynamics of two-electron systems in intense laser pulses, Phys. Rev. E 81, 056705 (2010).

[32] H. G. Muller, An efficient propagation scheme for the timedependent Schrödinger equation in the velocity gauge, Laser Phys. 9, 138 (1999).

[33] T. K. Kjeldsen, L. A. A. Nikolopoulos, and L. B. Madsen, Solving the m-mixing problem for the three-dimensional timedependent Schrödinger equation by rotations: Application to strong-field ionization of $\mathrm{H}_{2}^{+}$, Phys. Rev. A 75, 063427 (2007).

[34] T. E. Sharp, Potential-energy curves for molecular hydrogen and its ions 1, Atomic Data 2, 119 (1971).

[35] J. S. Sims and S. A. Hagstrom, High precision variational calculations for the Born-Oppenheimer energies of the ground state of the hydrogen molecule, J. Chem. Phys. 124, 094101 (2006).

[36] L. B. Madsen, L. A. A. Nikolopoulos, T. K. Kjeldsen, and J. Fernández, Extracting continuum information from $\Psi(t)$ in time-dependent wave-packet calculations, Phys. Rev. A 76, 063407 (2007).

[37] K. P. Huber and G. Herzberg, Molecular Spectra and Molecular Structure. IV. Constants of Diatomic Molecules (Van Nostrand Reinhold, New York, 1979).

[38] K.-F. Lai, V. Hermann, T. M. Trivikram, M. Diouf, M. Schlösser, W. Ubachs, and E. J. Salumbides, Precision measurement of the fundamental vibrational frequencies of tritiumbearing hydrogen molecules: $\mathrm{T}_{2}$, DT, HT, Phys. Chem. Chem. Phys. 22, 8973 (2020).

[39] D. C. Harris and M. D. Bertolucci, Symmetry and Spectroscopy: An Introduction to Vibrational and Electronic Spectroscopy (Oxford University Press, Oxford, 1978).
[40] I. Borges, Jr. and C. E. Bielschowsky, Doubly-excited states of molecular hydrogen: Theoretical absorption and photodissociation cross sections, J. Phys. B 33, 1713 (2000).

[41] T. Aoto, Y. Hikosaka, R. I. Hall, K. Ito, J. Fernández, and F. Martín, Dissociative photoionization of $\mathrm{H}_{2}$ at high photon energies: Uncovering new series of doubly excited states, Chem Phys. Lett. 389, 145 (2004).

[42] D. Pengel, S. Kerbstadt, D. Johannmeyer, L. Englert, T. Bayer, and M. Wollenhaupt, Electron Vortices in Femtosecond Multiphoton Ionization, Phys. Rev. Lett. 118, 053003 (2017).

[43] D. Pengel, S. Kerbstadt, L. Englert, T. Bayer, and M. Wollenhaupt, Control of three-dimensional electron vortices from femtosecond multiphoton ionization, Phys. Rev. A 96, 043426 (2017).

[44] S. Kerbstadt, K. Eickhoff, T. Bayer, and M. Wollenhaupt, Odd electron wave packets from cycloidal ultrashort laser fields, Nat. Commun. 10, 658 (2019).

[45] S. Kerbstadt, K. Eickhoff, T. Bayer, and M. Wollenhaupt, Control of free electron wave packets by polarization-tailored ultrashort bichromatic laser fields, Adv. Phys.: X 4, 1672583 (2019)

[46] A. Apalategui and A. Saenz, Multiphoton ionization of the hydrogen molecule $\mathrm{H}_{2}$, J. Phys. B 35, 1909 (2002).

[47] J. Ullrich and H. Schmidt-Böcking, Time-of-flight spectrometer for the determination of microradian projectile scattering angles in atomic collisions, Phys. Lett. A 125, 193 (1987).

[48] J. Ullrich, R. Moshammer, A. Dorn, R. Dörner, L. P. H. Schmidt and H. Schmidt-Böcking, Recoil-ion and electron momentum spectroscopy: Reaction-microscopes, Rep. Prog. Phys. 66, 1463 (2003).

[49] P.-C. Huang et al., Polarization control of isolated highharmonic pulses, Nat. Photonics 12, 349 (2018). 